\title{
Radio Emissions from Solar Active Regions
}

\author{
Jeongwoo Lee
}

Received: 15 February 2007 / Accepted: 4 May 2007 / Published online: 1 August 2007

\begin{abstract}
Solar active region coronae are known for strong magnetic fields permeating tenuous plasma, which makes them an ideal astronomical laboratory for magnetohydrodynamics research. It is, however, relatively less known that this physical condition also permits a very efficient radiation mechanism, gyro-resonant emission, produced by hot electrons gyrating in the coronal magnetic field. As a resonant mechanism, gyro-emission produces high enough opacity to fully reveal the coronal temperature, and is concentrated at a few harmonics of the local gyrofrequency to serve as an excellent indicator of the magnetic field. In addition, the polarization of the ubiquitous free-free emission and a phenomenon of depolarization due to mode coupling extend the magnetic field diagnostic to a wide range of coronal heights. The ability to measure the coronal temperature and magnetic field without the complications that arise in other radiative inversion problems is a particular advantage for the active region radio emissions available only at these wavelengths. This article reviews the efforts to understand these radiative processes, and use them as diagnostic tools to address a number of critical issues involved with active regions.
\end{abstract}

Keywords Sun: solar corona $\cdot$ Solar magnetic fields $\cdot$ Solar radio emission

\section{Introduction}

In the early days of radio astronomy, Covington (1947) observed a sharp decrease in the $10.7 \mathrm{~cm}$ flux during a lunar occultation of a large sunspot group, suggesting a connection between active regions and the radio flux from the Sun. It was of curiosity at that time which radiation mechanism could produce such a high opacity in the tenuous corona. Naturally, free-free emission from the ionized plasma in the corona was first suspected as a possible mechanism. Soon after, however, a large polarization was discovered (Covington 1949; Piddington and Minnett 1951) that could not be explained by free-free emission. It turned

J. Lee ( $₫)$

Physics Department, New Jersey Institute of Technology, University Heights, Newark, NJ 07102-1982,

USA

e-mail: leej@njit.edu 
out that gyro-resonant absorption can be a viable mechanism for the radio emission from sunspots (e.g., Zheleznyakov 1962; Kakinuma and Swarup 1962). High-resolution observations then revealed a close relationship of total intensity and polarization of radio emissions with magnetic field structure on the sun (e.g., Kundu et al. 1977; Alissandrakis and Kundu 1982). It is now established that the compact, bright, sunspot-associated sources can be attributed to gyro-resonant radiation (Lang and Willson 1982; Chiuderi-Drago et al. 1982; Shevgaonkar and Kundu 1985) while the extended, low brightness, and plage-associated sources arise from free-free emission (Chiuderi-Drago et al. 1977; Dulk and Gary 1983; Lang et al. 1983). According to the theory (Zlotnik 1968a, 1968b; Hurford 1986), solar gyro-resonant source should produce a spectrum that exhibits a constant brightness temperature up to some transition frequency, and then drops rapidly beyond that frequency, which has been confirmed in a series of multifrequency observations (Lee et al. 1993a, 1993b). Gary and Hurford (1994) presented a map of spatially resolved spectra that shows gyroresonant radiation in regions of strong magnetic field, and the free-free emission comes up in regions where the magnetic field is too weak for gyro-resonance.

Both types of radio emissions are polarized due to the presence of magnetic field in the corona above an active region. The polarization of free-free emission could be used to determine the line-of-sight component of the coronal magnetic field (Bogod and Gelfreikh 1980). On the other hand, gyro-resonant emission is, in general, very optically-thick and otherwise too weak to be used for polarization measurement. Rather, due to the fact that strong opacity is limited to a few harmonics of gyrofrequency, it can be used for direct measurement of total magnetic field strength (Gelfreikh and Nefedev 1975). Another interesting phenomenon is the conversion of polarized radio emission into unpolarized emission when passing through a region of transverse magnetic field (Cohen 1960). The efficiency of mode coupling strongly depends on both density and magnetic field in the region, independent of the source radiation mechanism. The depolarization phenomenon and free-free emission occur in a wide range of height in the corona depending on frequency (up to $\sim 10^{4} \mathrm{~km}$ ), and they thus complement the magnetic field diagnostic of the gyro-resonant mechanism dominant in relatively low $\left(\sim 10^{3} \mathrm{~km}\right)$ and strong field $\left(10^{2}-10^{3}\right.$ Gauss $)$ regions.

The presence of the efficient radio emission from the corona is important for many reasons. The first is that the corona is generally an object that is too thin to be detected by ground-based observations. It is the gyration and collisions of hot electrons in the magnetized medium that produces the radiative power sufficient to be detected on the ground at radio frequencies. At radio wavelengths, imaging can be done with interferometric techniques, and arrays such as the Westerbork Synthesis Radio Telescope (WSRT), the Very Large Array (VLA), Nobeyama Radioheliograph (NoRH), and the Siberian Solar Radio Telescope (SSRT) provide adequate spatial resolution for active region studies at a few fixed frequencies. For spectroscopy, one needs to cover a wide frequency range (e.g., 1-20 GHz) at high spectral resolution since interesting spectral features can occur in any of these frequencies. This capability is provided by frequency-agile radiotelescopes: the Owens Valley Solar Array (OVSA) and RATAN 600. From the diagnostic viewpoint, it is attractive that these radiations allow direct measurement of temperature and magnetic field without the complications that arise from analysis at other wavelengths; for example, free from chemical abundance and filling factor dependence. Furthermore, the outputs of these radiations are, in fact, the very target quantities needed to address the physics of the corona. On the other hand, the corona can be observed at other wavelengths with space-borne instruments: X-ray wavelengths with Yohkoh/SXT and extreme-ultraviolet (EUV) with instruments aboard the Solar and Heliospheric Observatory (SOHO) satellite (Domingo et al. 1995), and the Transition Region and Coronal Explorer (TRACE). These images reveal a highly 
filamentary structure of the corona, which presents a new challenge in solar physics issues where the magnetic connectivity plays an important role. It is therefore highly desirable to combine the distinct properties of radio emission with the information obtained at EUV and $\mathrm{X}$-ray wavelengths to provide further insight into the coronal physics.

Many review papers have been published on the subject of active region radio emissions (Zlotnik 1968a, 1968b; Zheleznyakov 1970; Lantos 1972; Gelfreikh and Lubyshev 1979; Vourlidas 1996; White and Kundu 1997) and the most comprehensive papers can be found in a recent book dedicated to solar and space weather radiophysics (Gary and Keller 2004). A motivation for this review is that the presently known and potential radio capabilities should be updated with the recent contribution from space-borne imaging at EUV and $\mathrm{X}$-rays. The plan of this paper is as follows: Sect. 2 presents some elements of the theoretical backgrounds for the radiation processes. Section 3 describes relevant observations. Section 4 briefly discusses scientific issues of the active region corona that can be addressed by the radio observations, and Sect. 5 concludes the review and suggests future directions for radio studies.

\section{Theoretical Background}

We start with the elementary radiative properties of active region radio emissions that do not depend on specific radiation mechanisms (Sect. 2.1) and then go through specific mechanisms (Sects. 2.2 and 2.3).

\subsection{Basic Properties of Active Region Emission}

\subsubsection{Particles and Waves}

Since the gyro-resonance is basically a type of wave-particle interaction, we should deal with the evolution of particles and waves under mutual interactions. This is generally a complex issue, but under solar coronal conditions a much simpler approximation called magneto-ionic theory (Hartree 1931; Appleton 1932) is applicable. The magneto-ionic theory has historically been developed for the radio wave propagation in the ionosphere, in which the particles, electrons and ions, are thermal and too cold to affect the wave motion. The particle property is thus simply given by the Maxwellian velocity, which is compared with the phase velocity of the plasma wave $v_{\phi}=c / n_{\mathrm{r}}$. Here $c$ is the speed of light and $n_{\mathrm{r}}$ is the index of refraction. $n_{\mathrm{r}}$ is expressed as a function of radio frequency $v$ and angle $\theta$ between the wave propagation and the local magnetic field for a given plasma characterized by the gyrofrequency $v_{B}$ and the plasma frequency $v_{\mathrm{p}}$ :

$$
v_{B}=\frac{e B}{2 \pi m_{\mathrm{e}} c}, \quad v_{\mathrm{p}}=\sqrt{\frac{n_{\mathrm{e}} e^{2}}{\pi m_{\mathrm{e}}}},
$$

where $m_{\mathrm{e}}$ and $e$ are the mass and electric charge of an electron, respectively; $n_{\mathrm{e}}$ is the number density of electrons; and $B$ is the magnetic field strength in Gauss. Throughout this paper, all quantities are given in the cgs-Gaussian units. When this refractive index is plotted as a function of frequency for a given density and magnetic field, it reveals four branches of possible wave modes: the ordinary (O mode), extraordinary (X mode), whistler and Z-modes. The latter two modes cannot propagate through vacuum, and those generated in the Sun can never be observed from Earth. Only the whistler and $\mathrm{Z}$ mode waves generated near the Earth have been found through in situ observations with satellites. The $\mathrm{X}$ and $\mathrm{O}$ 
modes can possibly escape the Sun and reach observers at Earth, and they are the solar radio emissions to be discussed in this paper. The refractive indices for the $\mathrm{X}$ and $\mathrm{O}$ modes are given by (see Stix 1962)

$$
n_{\mathrm{X}, \mathrm{O}}^{2} \approx 1-\frac{2 w v_{\mathrm{p}}^{2}}{2 w v^{2}-v_{B}^{2} \sin ^{2} \theta \mp \sqrt{v_{B}^{4} \sin ^{4} \theta+4 w^{2} v_{B}^{2} \nu^{2} \cos ^{2} \theta}},
$$

where $w \equiv 1-v_{\mathrm{p}}^{2} / v^{2}$, and the negative (positive) sign in front of the square root term is $\mathrm{X}$ (O) mode. One can see that in an unmagnetized plasma, the index of refraction degenerates to $n_{\mathrm{r}}^{2} \approx w$. The $\mathrm{X}$ mode is polarized in the same sense that the electron gyrates about the magnetic field, and interacts more strongly with electrons than the $\mathrm{O}$ mode. As a result the emission is generally polarized in $\mathrm{X}$ mode upon generation, although an exception occurs at a very low value of $\theta$.

\subsubsection{Possible Frequencies}

The lowest frequencies of the $\mathrm{X}$ and $\mathrm{O}$ mode waves are set by the requirement that $n_{\mathrm{X}, \mathrm{O}}^{2} \geq 0$. These are called cut-off frequencies and are given by

$$
v_{\mathrm{O}} \geq v_{\mathrm{p}}, \quad v_{\mathrm{X}} \geq \frac{v_{B}}{2}+\sqrt{v_{\mathrm{p}}^{2}+\frac{v_{B}^{2}}{4}}
$$

for all range of $\theta$. Under typical active region coronal conditions, $B \sim 100-1000$ Gauss and $n_{\mathrm{e}}=10^{9} \mathrm{~cm}^{-3}$, these frequencies of the active region radio emission come out of order of $1 \mathrm{GHz}$. In principle there is no limit in the high frequency, but in practice, we may set it to the turnover frequency where the opacity $\tau$ becomes unity since the flux will diminish significantly above that frequency. Under the typical parameter values, the turnover frequency for gyro-resonant radiation (9) is found to be at the third to fifth harmonic of local gyrofrequency, and that frequency for free-free emission (11) depends largely on the electron emission measure, but usually lies below $\sim 20 \mathrm{GHz}$. Thus, the active region radio emissions reside primarily in the microwave frequency range, which is fortunate because in this frequency range spectroscopy and imaging are available at reasonable resolutions. It is also worthwhile to note that the frequency range of radio emission will be lowered at greater heights as magnetic field and plasma density decrease with heights (see Fig. 4.1 of Gary and Hurford 2004). The observing frequency appropriate to the coronal height of interest may therefore be chosen to some extent.

\subsubsection{Radiative Transfer}

The properties of a radiation field are not fixed when it is created. It will continue to be modified by the local medium as it propagates to the observer. This is known as the radiative transfer problem. Radiative transfer is generally described in terms of specific intensity, $I_{v}$, defined as the radiation power per unit frequency and per unit solid angle crossing a unit area, and the transfer equation is in the form:

$$
n_{\mathrm{r}}^{2} \frac{\mathrm{d}}{\mathrm{d} l}\left[\frac{I_{v}}{n_{\mathrm{r}}^{2}}\right]=-\kappa_{v} I_{v}+j_{v}
$$

where $\kappa_{v}$ is the absorption coefficient, $j_{v}$ is the volume emissivity, and $\mathrm{d} l$ is the element of the ray path. We usually adopt the approximation $n_{\mathrm{r}} \approx 1$, valid at high frequencies, which 
also means that the ray path is a straight line from the source to the observer. In the present case, it is possible and also preferable to express the transfer equation in units of temperature rather than intensity (Kundu 1965; Rybicki and Lightman 1979). First, we divide each term by $\kappa_{v}$ and identify the ratio $j_{v} / \kappa_{v}$ with the source function. For thermal radiation, the source function is given by the Planck function $B_{v}\left(T_{\mathrm{e}}\right)$ of the local electron temperature $T_{\mathrm{e}}$ (called Kirchhoff's law). Since the source term mainly depends on electron temperature, it is useful to express the specific intensity in terms of a temperature. The concept of brightness temperature, $T_{\mathrm{b}}$, is then introduced, defined by the temperature of the blackbody having the same intensity at that frequency, that is, $I_{v} \equiv B_{v}\left(T_{\mathrm{b}}\right)$. At the relevant wavelengths, the RayleighJeans law applies and the source function can be approximated by $B_{v}\left(T_{\mathrm{e}}\right) \approx 2 k v^{2} T_{\mathrm{e}} / c^{2}$, and the specific intensity, $I_{v} \approx 2 k v^{2} T_{\mathrm{b}} / c^{2}$ where $k$ is the Boltzmann's constant. We can thus rewrite the transfer equation in terms of brightness temperature as follows:

$$
\frac{\mathrm{d} T_{\mathrm{b}}}{\mathrm{d} \tau}=-T_{\mathrm{b}}+T_{\mathrm{e}},
$$

where $\tau \equiv \int \kappa \mathrm{d} l$ is the optical depth or opacity. This equation has a formal solution:

$$
T_{\mathrm{b}}\left(\tau_{\mathrm{m}}\right)=\int_{0}^{\tau_{\mathrm{m}}} T_{\mathrm{e}}(\tau) \mathrm{e}^{-\tau} \mathrm{d} \tau+T_{\mathrm{b}}(0) \mathrm{e}^{-\tau_{\mathrm{m}}},
$$

where $\tau_{\mathrm{m}}$ refers to the maximum opacity along a given line of sight. For an optically thick source $\tau_{\mathrm{m}}$ is effectively infinity, and $T_{\mathrm{b}}(\infty)=\int_{0}^{\infty} T_{\mathrm{e}}(\tau) \mathrm{e}^{-\tau} \mathrm{d} \tau \approx T_{\mathrm{e}}$ at $\tau \approx 1$. It is thus easy to see, in this form, how the observed radio brightness temperature is related to the local coronal electron temperature. We have not yet distinguished the polarization states of the radiation in the transfer equation, merely for convenience. When we define the brightness temperature, $2 T_{\mathrm{b}}$ should, in fact, be the sum of $T_{\mathrm{X}}$ and $T_{\mathrm{O}}$, and likewise, $2 T_{\mathrm{e}}$ in the source function is the sum of equal contributions to each polarized mode. Thus, (5) and (6) remain valid as long as we substitute $\left(T_{\mathrm{b}}, \tau\right)$ with $\left(T_{\mathrm{X}}, \tau_{\mathrm{X}}\right)$ for the $\mathrm{X}$ mode and with $\left(T_{\mathrm{O}}, \tau_{\mathrm{O}}\right)$ for the O mode, respectively.

\subsubsection{Polarizations}

Although the generation of two natural modes, $T_{\mathrm{X}}$ and $T_{\mathrm{O}}$, proceeds independently as above, we observe them at the Earth in terms of right- and left-circular polarized (RCP and LCP) brightness, $T_{\mathrm{R}}$ and $T_{\mathrm{L}}$, respectively. At each point along the ray path, $T_{\mathrm{R}, \mathrm{L}}$ may be decomposed into a sum of components in the two local natural modes of the plasma, $T_{\mathrm{X}, \mathrm{O}}$, and the full treatment of the polarization is complicated (see the following). Without the detailed knowledge of the polarization, we may infer that when the magnetic field points toward us along the line of sight, we will see perfectly circular polarization with $T_{\mathrm{R}}=T_{\mathrm{X}}$ and $T_{\mathrm{L}}=T_{\mathrm{O}}$ and vice versa depending on the magnetic polarity. If the magnetic field is perpendicular to the line of sight, they will be completely linearly polarized so that $T_{\mathrm{R}}=T_{\mathrm{L}}=\left(T_{\mathrm{X}}+T_{\mathrm{O}}\right) / 2$. This is correct but, for a quantitative study, we need to know exactly at which angle the transition from the circular to linear polarization occurs.

The general relationship between the circular polarization and the natural modes was given by (Papagianis and Kogut 1975; Vourlidas 1996)

$$
T_{\mathrm{R}, \mathrm{L}}=\frac{1}{2}\left(T_{\mathrm{X}}+T_{\mathrm{O}}\right) \pm \frac{r}{1+r^{2}}\left(T_{\mathrm{X}}-T_{\mathrm{O}}\right)
$$


where $+/-$ corresponds to RCP/LCP states, respectively, for the positive magnetic polarity. $r$ is the ratio of the electric fields, $E_{x}$ and $E_{y}$, in the axes orthogonal to the ray path set to the $z$-axis, and is expressed by (Ratcliffe 1959)

$$
r \equiv \frac{E_{x}}{E_{y}}=q+\sqrt{1+q^{2}}, \quad q=\frac{v_{B} / 2 v}{1-\left(v_{\mathrm{p}} / v\right)^{2}} \frac{\sin ^{2} \theta}{\cos \theta} .
$$

These parameters determine the polarization of the natural modes in the plasma, that is, whether they are circularly polarized $(r=1, q \ll 1)$ or linearly polarized $(r, q \gg 1)$ at a given position along the ray path. We can see that the factor $q$ depends on not only $\theta$ but also on $v_{\mathrm{p}}$ (density) and $v_{B}$ (magnetic field strength). As far as $v \gg v_{B}$, $v_{\mathrm{p}}$, we have $q \ll 1$ for most angles except $\theta \approx 90^{\circ}$. Therefore the solar radiation is generally circularly polarized when emitted, and the two natural modes are in most cases circularly polarized everywhere except for a narrow range of propagation angles orthogonal to the local magnetic field.

\subsection{Emission Mechanisms}

We have not yet discussed the values of $T_{\mathrm{X}}$ and $T_{\mathrm{O}}$ in the two radiation mechanisms, gyroresonant and free-free emission. Radiation theory typically involves calculation of the emissivity and absorption coefficient. As noted earlier, these two quantities are, however, related to each other for thermal radiations under the Kirchhoff's law. It is therefore both convenient and complete to deal with only one of these two quantities. Here, we give expressions for the line-of-sight integral of absorption coefficient, that is, opacity.

\subsubsection{Gyro-resonant Emission}

The gyro-resonant opacity is calculated from the interaction of plasma waves and electrons gyrating around magnetic fields. The resulting emission is, as expected, sharply peaked at each harmonic frequency, $v=s v_{B}$ where $s$ is the harmonic number, and the spectral width of a cyclotron resonance is proportional to $\mu^{-1 / 2} s v_{B}$ where $\mu=m_{\mathrm{e}} c^{2} / k_{\mathrm{B}} T \gg 1$ (see, e.g., Melrose 1980; Dulk 1985). Since the emission (or, equivalently, the absorption) rapidly decreases at frequencies away from the resonant frequency $v=s v_{B}$, it is customary to use, for convenience, an alternative expression for the absorption coefficient that is averaged over the profile of an individual harmonic. This average over the frequency results in an expression that contains a magnetic scale length $L_{B}=B / \nabla B$. The final form of the gyroresonant opacity is given by

$$
\tau^{\mathrm{g}-\mathrm{r}}(s, v, \theta) \approx 0.0133 \frac{n_{\mathrm{e}}}{v} \frac{s^{2}}{s !}\left(\frac{s^{2} \sin ^{2} \theta}{2 \mu}\right)^{s-1} L_{B} F_{\mathrm{X}, \mathrm{O}}(\theta),
$$

where the complicated angle dependence is separated out into the function $F_{\mathrm{X}, \mathrm{O}}$. In many cases, the approximation $F_{\mathrm{X}, \mathrm{O}} \approx(1 \pm \cos \theta)^{2}$ is used, where the positive (negative) sign is for the $\mathrm{X}(\mathrm{O})$ mode. This approximation is, however, good only at angles away from $90^{\circ}$, and should not be taken to imply $\tau_{\mathrm{X}} \approx \tau_{\mathrm{O}}$ at $\theta \approx 90^{\circ}$. In fact, at angles near $90^{\circ} F_{\mathrm{X}} \approx 1$ and $F_{\mathrm{O}} \approx \mu^{-1}$, and therefore the $\mathrm{O}$ mode opacity is always smaller than the $\mathrm{X}$ mode opacity at all angles. Accurate expressions for these opacities that correctly handle the mode polarization properties were presented by Zlotnik (1968a), and were plotted by White and Kundu (1997).

From expression (9) alone, it is not so obvious where the sensitivity of the opacity to magnetic field strength lies. Since the resonant condition is already incorporated into this 
expression, it is implicit that the opacity should apply only to a point where the resonance condition is met. A proper way to represent this resonant opacity would be

$$
\tau^{\mathrm{g}-\mathrm{r}}(v, \theta)=\sum_{s=1} \tau^{\mathrm{g}-\mathrm{r}}(s, v, \theta) \delta\left(B-\frac{B_{0}}{s}\right),
$$

so that the opacity is evaluated only in the region satisfying the resonance condition of $B=B_{0} / s$ and set to zero elsewhere. Here $B_{0}[\mathrm{Gauss}]=357 v[\mathrm{GHz}]$. An advantage of gyroresonant opacity is obvious in view of (6) and (10). Since the opacity is either (effectively) infinitely large or negligibly small, the solution for $T_{\mathrm{X}}$ or $T_{\mathrm{O}}$ often ends up with the electron temperature at the last (greatest in height) optically thick gyro-resonant layers at each mode. The gyro-resonant layer corresponding to a lower frequency will lie progressively higher in the corona, which leads to the statement: "the coronal magnetic structure can be peeled away by using different frequencies" (White 2004).

\subsubsection{Free-free emission}

Free-free emission is produced by the collisions of electrons with protons and other particles, and must be emitted from the corona, which is composed of fully ionized plasma. The line-of-sight integration is straightforward in this case, and the free-free opacity is given by

$$
\tau^{\mathrm{f}-\mathrm{f}}(\nu, \theta) \approx \frac{9.8 \times 10^{-3}}{\left(\nu \mp v_{B}|\cos \theta|\right)^{2}} \frac{n_{\mathrm{e}}^{2} L_{\rho}}{T_{\mathrm{e}}^{1.5}},
$$

where $L_{\rho}$ is the thickness of the plasma containing an average density $n_{\mathrm{e}}$ at temperature $T_{\mathrm{e}}$ and all quantities here are given in c.g.s. units. The negative (positive) sign in the denominator corresponds to $\mathrm{X}(\mathrm{O})$ mode. This approximation is valid when $v \gg v_{B}, v_{\mathrm{p}}$. The latter condition is naturally guaranteed because propagating waves must have a frequency greater than $v_{\mathrm{p}}$ and, at frequencies close to low harmonics of $v_{B}$, the free-free emission is unimportant anyway due to the dominant gyro-resonant emission.

For free-free emissions, the degree of circular polarization can be determined easily and it depends only on the line-of-sight magnetic field and frequency as

$$
\frac{\left|T_{\mathrm{R}}-T_{\mathrm{L}}\right|}{T_{\mathrm{R}}+T_{\mathrm{L}}} \approx \frac{T_{\mathrm{X}}-T_{\mathrm{O}}}{T_{\mathrm{X}}+T_{\mathrm{O}}} \approx \frac{\tau_{\mathrm{X}}-\tau_{\mathrm{O}}}{\tau_{\mathrm{X}}+\tau_{\mathrm{O}}} \approx 2 \frac{v_{B}}{v}|\cos \theta| .
$$

This is an example of the general rule that the optically thin polarization can be used to determine the magnetic field responsible for the polarization.

It is tempting to write an expression for $\left(\tau_{X}-\tau_{O}\right) /\left(\tau_{X}+\tau_{O}\right)$ expected under gyroresonant emission as well. This ratio is, however, not equivalent to the observed degree of circular polarization, because it is rarely the case under gyro-resonant radiation that both $\mathrm{X}$ and $\mathrm{O}$ modes are observed to be optically thin simultaneously. More often than not, the $\mathrm{X}$ mode is optically thick while the $\mathrm{O}$ mode is thin in the same harmonic layer; otherwise, both modes tend to be very thick or very thin. These extreme behaviors arise because the gyroresonant opacities are generally very large at the lowest harmonic and then rapidly drop with increasing harmonic. As far as gyro-resonant radiation is concerned, it is generally preferable to use the $\mathrm{X}$ and $\mathrm{O}$ modes separately for magnetic field information. 


\subsection{Mode Coupling}

We noted above that the $\mathrm{X}$ and $\mathrm{O}$ modes propagate independently of each other in general. An exception to this rule may happen when they enter a region where the line-of-sight component of magnetic field reverses, that is, the angle $\theta$ goes through $90^{\circ}$, called a quasitransverse (QT) layer. After passing the QT layer, the wave vector has to reverse its sense of rotation in order to remain in the original natural mode ( $\mathrm{X}$ or $\mathrm{O}$ mode). Like many other physical quantities, the wave vector prefers to maintain its physical state, that is, the sense of rotation in this case. However, nature does not always allow this since the two natural modes are quite clearly circular. An exception can therefore happen when the mode property rapidly changes from circular $(q \ll 1)$ to linear $(q \gg 1)$ polarization within the QT layer and this strongly couples the two modes to each other such that their identities are exchanged within the layer. This situation is theoretically expressed as (Zheleznyakov and Zlotnik 1964; Melrose 1985)

$$
V_{\text {obs }}=V_{\text {int }}\left(2 \mathrm{e}^{-\pi / 2 Q}-1\right) \text {, }
$$

where $V_{\mathrm{obs}}$ and $V_{\text {int }}$ are, respectively, the observed and intrinsic degree of circular polarization (i.e., in terms of $T_{\mathrm{R}, \mathrm{L}}$, not $T_{\mathrm{X}, \mathrm{O}}$ ) and $Q$ is called mode coupling coefficient. Note that for strong coupling $(Q \gg 1)$, the mode identity changes, but this means that the apparent polarization $V$ does not change, because $\mathrm{X}$ mode before entering the QT layer is now O-mode after passing through the QT layer, and this $\mathrm{O}$ mode in the new environment still has the same sense of rotation relative to the line of sight. In case of weak coupling $(Q \ll 1)$ the apparent polarization $V$ should reverse as the polarity of ambient magnetic field changes after passing the QT layer while the mode identity is maintained. For intermediate values of $Q$ the observed polarization is reduced from the original one by the factor given by (13).

The coupling parameter $Q$ is determined by the rate at which the two modes become coupled relative to the rate at which they get out of phase (Melrose 1985). The latter is simply given by the difference between the refractive indices of the $\mathrm{X}$ and $\mathrm{O}$ modes (2). The former concerns how rapidly the modes change from circular to linear polarization, that is, how fast $q$ approaches to zero. In view of the expression for $q$ (8), there are three ways that this can happen: (i) when $\theta$ is passing through $90^{\circ}$ (Cohen 1960); (ii) when a fine-scale density fluctuation exists such that $v \approx v_{\mathrm{p}}$ (Melrose 1975); and (iii) when there is a magnetic-field-free region inside the QT layer so that the modes instantly lose their identity to degeneracy (Zheleznyakov et al. 1996; Zheleznyakov and Zlotnik 1988). We can see that the last two scenarios require special conditions on density or magnetic field that are possible but unlikely. The first condition, on the other hand, has to occur anyway when the ray passes through the QT layer. Furthermore, the variation of $q$ around $\theta=90^{\circ}$ can be very rapid even though the density and magnetic field are slowly varying, as is likely for solar corona. Therefore, the first scenario has mostly been assumed in studies of mode coupling phenomena, in which case $Q$ is given by (Cohen 1960; Bandiera 1982; Melrose 1985)

$$
Q \approx 0.2 v^{4} v_{B}^{-3} v_{\mathrm{p}}^{-2} L_{\theta}^{-1},
$$

where $L_{\theta}$ is the scale length for the viewing angle variation in units of $\mathrm{km}$, and all frequencies here are in $\mathrm{MHz}$. Typically this expression is applied to the depolarization condition, $Q=\pi / 2 \ln 2$, to derive either a density or magnetic field strength that pertains to the region of the depolarization strip in the QT layer. 


\subsection{Modeling of Active Region Radio Emissions}

For a direct comparison of the theory with solar radio observations, it is generally necessary to calculate theoretical radio maps or spectra by applying the basic physics to a realistic model atmosphere. Specifically one needs to calculate theoretical opacities (9-11) using the magnetic field, temperature and density specified in a model atmosphere, and perform the line-of-sight integration (6) to determine the brightness temperature as a function of either spatial position on the projected sky plane or observing frequency and polarization. Depending on the magnetic field configuration, mode coupling $(13,14)$ may also need to be calculated.

The efforts of modeling radio emissions may be classified into two categories. In the first category, model calculations are carried out to understand general properties of active region radio emissions expected under the theory. They typically adopt generic magnetic field models (e.g., dipole field) in calculation of theoretical radio emissions either as 1D profiles (Zlotnik 1968a, 1968b; Holman and Kundu 1985; Vourlidas et al. 1997; White and Kundu 1997) or 2D maps (Gelfreikh and Lubyshev 1979; Brosius and Holman 1989; Lee et al. 1993b). The results illustrate how the radio morphology varies with the observing frequency, viewing angle and polarization state and can thus guide the observing plan and interpretation of the results. Recently, Gary et al. (2007) carried out a simulation of realistic radio maps to evaluate the diagnostic capability of radio observations. They calculated brightness temperature using a fully 3D model of temperature, density and magnetic field (Mok et al. 2005) and passed the result through a model instrument with realistic noise and sensitivity. They then tested whether the diagnostic tools offered by gyro-resonant and free-free emission mechanisms can really recover the original model atmosphere. Some of the test results are presented in Gary and Keller (2004, their Figs. 4.3, 4.5 and 5.10) and are promising. Similarly, Aschwanden et al. (2004) tested the diagnostics of free-free emissions in weak field regions, using comparison with EUV and soft X-rays.

The second category includes all modeling performed to assist interpretations or analyses of specific observations. They usually use coronal magnetic fields reconstructed from the photospheric magnetograms via a technique called coronal field extrapolation (see Sect. 4.2). Since Alissandrakis and Kundu (1984) first used such a field extrapolation model to interpret their observed radio morphology, this type of modeling has become more and more popular and a variety of techniques have appeared. To list a few, Alissandrakis et al. (1996) and Lee et al. (1998b) calculated a variety of different field extrapolation models, and chose the most plausible one based on its ability to explain the observed mode coupling phenomenon. Lee et al. (1998a, 1999a, 1999b) performed model fitting to VLA observations to determine the electron temperature structure with a well-tested magnetic field model. Most of these works are, by nature, forward-fitting modeling and thus the choice of free model parameters can be an issue. In some approaches, EUV or soft X-ray data are used to deduce temperature and density to reduce the free parameters of the model atmosphere so that magnetic field information can finally be derived from radio observations (e.g., Brosius et al. 1993, 1997, 2002).

\section{Observations}

Observations of active region radio emissions have been carried out in either Fouriertransform imaging or broadband spectroscopy or both, including both circular polarizations. We organize the numerous observational results into selective categories, and describe how they are currently understood. 

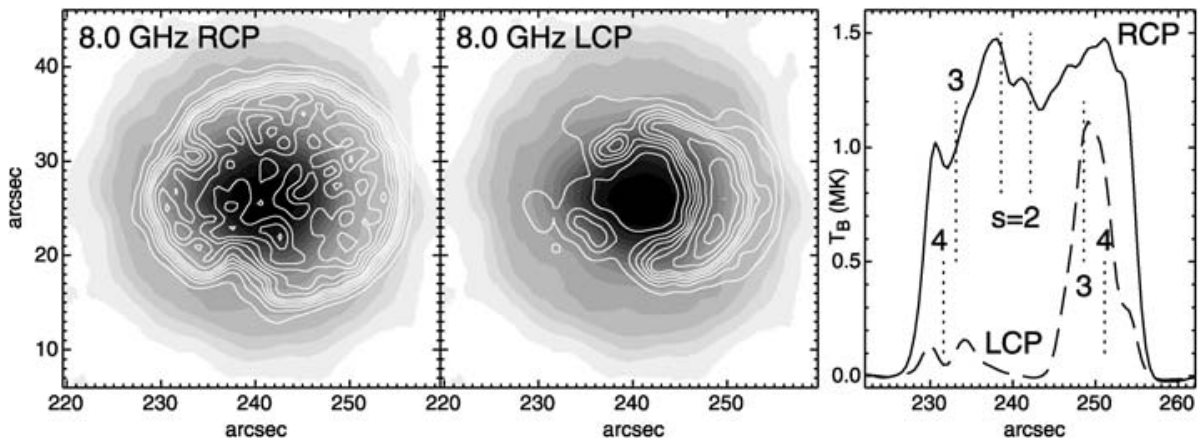

Fig. 1 VLA observations of a sunspot on July 17, 2001, at 8.0 GHz. Left panel: contours of RCP (X mode for this spot) brightness superimposed on a white light image. Middle panel: contours of LCP (O mode) brightness on the white light image. In both panels, contours are at brightness temperatures of 0.05 , $0.15, \ldots, 0.55,0.75,0.95, \ldots, 1.75 \times 10^{6} \mathrm{~K}$, and the resolution is $2^{\prime \prime}$. The X mode is optically thick everywhere, while the $\mathrm{O}$ mode shows the classic ring structure. Right panel: horizontal profiles (units of $10^{6} \mathrm{~K}$ ) through the radio images at a vertical coordinate of $25^{\prime \prime}$ for RCP (solid line) and LCP (dashed line), showing the presence of a number of sharp edges (from White 2004)

\subsection{Characteristic Morphologies of Isolated Sunspots}

Since the early days of solar radio imaging it has been known that radio sunspots appear in a shape of "horseshoe" or "ring" in polarization maps (Alissandrakis et al. 1980; Alissandrakis and Kundu 1982, 1984; Lang and Willson 1982; Kundu and Alissandrakis 1984; Vourlidas and Bastian 1996; Vourlidas et al. 1996). Figure 1 shows a recent observation of such a pattern obtained from a round sunspot with the VLA by White (2004). In LCP the map appears as a classical horseshoe shape. In RCP it shows a somewhat complicated structure within a circular disk outlining the optical sunspot, which at a lower resolution would appear as a classical disk structure.

These characteristics can be understood as either the presence of cool plasma $\left(T_{\mathrm{e}} \sim\right.$ $2 \times 10^{5} \mathrm{~K}$ ) overlying the umbra reported in EUV observations (e.g., Foukal 1976) or as dependence of the optical depth on the viewing angle (9) of a gyro-resonance source. The latter interpretation was presented by Vourlidas et al. (1997) after observations of a highly polarized sunspot emission over consecutive days. On the other hand, Zlotnik et al. $(1996,1998)$ analyzed the VLA observations of a sunspot appearing in a classical ring structure, but concluded that there must be cool dense plasma in the center of the sunspot, based on the sizes of the depression in $\mathrm{X}$ and $\mathrm{O}$ modes. If the depression is due to the angle effect, the size of the depression should be much smaller in the $\mathrm{X}$ mode than in the $\mathrm{O}$ mode, but they are observed to have comparable sizes. More recently, Brosius and White (2004) also observed the radio depression above an active region and interpreted it as evidence for the presence of cool plasma in the umbral corona.

\subsection{Frequency-dependent Morphology}

Without exception, the overall area of radio sources over an active region reduces with increasing frequency. Figure 2 shows VLA observations of a complex active region where we can see this behavior. Although the emission source at the middle frequency $(8.4 \mathrm{GHz})$ almost agrees with the optical active region in apparent size, the source at a lower frequency $(5 \mathrm{GHz})$ extends outside the optical active region, and at a higher frequency $(15 \mathrm{GHz})$ 


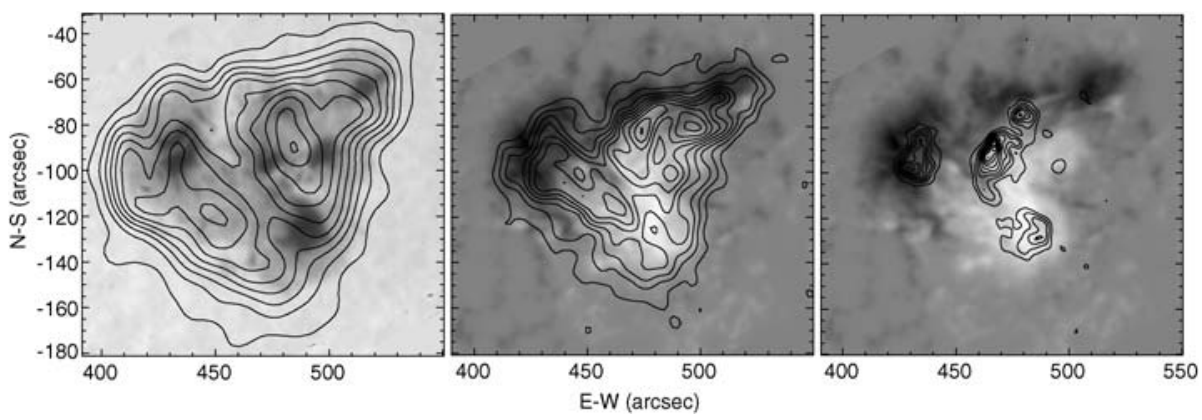

Fig. 2 VLA observations of a complex solar active region, AR 6615. The left panel shows the contours of the $5 \mathrm{GHz}$ emission overlaid on the white-light image showing a number of spots within the same penumbra. Due to unusually high brightness temperatures ( $\geq 6 \mathrm{MK}$ ), it is assumed that the radiation comes from the fourth harmonic layer, $s=4$. The gyro-resonant emission at $5 \mathrm{GHz}$ then corresponds to the electron temperature distribution on the surface in the corona where the magnetic field $B$ equals 450 Gauss. The middle and right panels show contours of the $8.4 \mathrm{GHz}$ ( $B=750 \mathrm{Gauss}$ ) and $15 \mathrm{GHz}$ emission ( $B=1350 \mathrm{Gauss}$ ), respectively, overlaid on a longitudinal magnetogram which indicates the direction of the magnetic field in the photosphere ( white $=$ upgoing, black $=$ downgoing field). The maximum brightness temperatures in the radio images are $4.4 \times 10^{6} \mathrm{~K}$ at $4.9 \mathrm{GHz}, 4.6 \times 10^{6} \mathrm{~K}$ at $8.4 \mathrm{GHz}$, and $1.8 \times 10^{6}$ at $15 \mathrm{GHz}$, respectively. Contours begin at $10 \%$ of the maximum brightness temperature and then are $10 \%$ apart (from Lee et al. 1997)

shrinks to smaller regions of strong magnetic fields. This frequency-dependent morphological change can be understood if the dominant radiation mechanism is an optically thick gyro-resonant emission, since the magnetic field strength required for gyro-resonance increases with frequency (see (10)). Of course, free-free emission always co-exists with gyroresonant emission and does not necessarily follow the same trend in morphology. However, the free-free emission is often too dim to modify the morphology due to the dominance of the gyro-resonant source and the finite dynamic range of the map. The frequency-dependent morphology, such as shown in Fig. 2, is therefore a strong piece of evidence for the dominance of gyro-resonant radiation.

Under this interpretation, the frequency-dependent morphology can be used to derive the spatial distribution of magnetic field strength. At the boundary of the radio source at each frequency, the emission changes from optically thick $(\tau \gg 1)$ to thin $(\tau \ll 1)$ spectrum, and therefore that observing frequency is the turn-over frequency of the local spectrum at that location. Thus, the local magnetic field strength corresponding to the observing frequency $v$ and the known effective harmonic can be derived from $B=357 v / s$, where $v$ is in units of $\mathrm{GHz}$ and $B$ is in Gauss. In this inversion we are using only the fact that the emission boundary is the place where the observed radio brightness temperature falls from the coronal temperature $\sim 10^{6} \mathrm{~K}$ to the chromospheric temperature $\sim 10^{4} \mathrm{~K}$, without concerning ourselves with the exact temperature and density. By repeating this procedure over many frequencies we can derive the distribution of field strength at the coronal base. Lee et al. (1993a) applied this technique to a round sunspot to determine the radial distribution of magnetic field above a sunspot.

\subsection{Spatially Resolved Spectrum}

Instead of checking the morphology change with frequency, one can construct a data-cube of 2D maps along the frequency axis, and then build a set of spectra at each common spatial point. This is called spatially resolved spectroscopy or imaging spectroscopy. Figure 3 


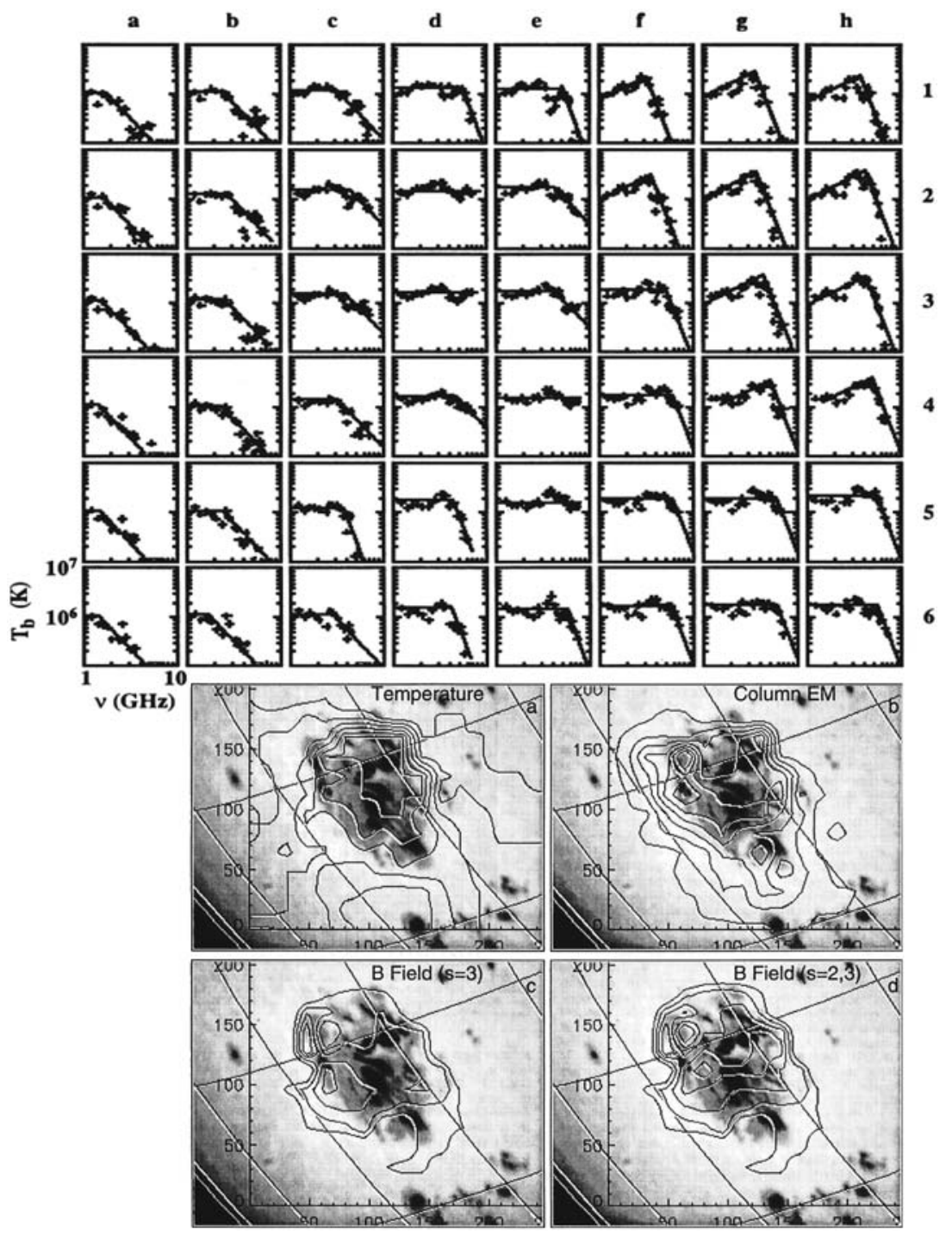

Fig. 3 Physical maps from the OVSA imaging spectroscopy. Top: Brightness temperature spectra obtained at 48 grid points in the data cube represented by the maps. The axis scales for the spectra are all the same, as shown on the spectrum at the lower left. Bottom: Physical maps obtained by interpretation of brightness temperature spectra. a Electron temperature with contour levels 0.9, 1.15, 1.4, 1.9 and $2.09 \times 10^{6} \mathrm{~K}$. b Electron column emission measure. c Total magnetic strength as measured from gyro-resonant spectra assuming harmonic $s=3$ everywhere. The contour levels are 200, 400, 600, 800 and 1000 Gauss. $\mathbf{d}$ The same as in $c$ except $s=2$ has been assumed in upper right part of the active region. The highest contour is, in this case, 1200 Gauss (from Gary and Hurford 1994)

shows the first demonstration of such imaging spectroscopy by Gary and Hurford (1994) in which the optically thick gyro-resonant layer corresponding to a given frequency and mode drops below the corona, the radio brightness temperature at that frequency and corresponding polarization shows a sharp drop from coronal $\left(\sim 10^{6} \mathrm{~K}\right)$ to approximately chromospheric 
values $\left(\sim 10^{4} \mathrm{~K}\right)$. The regions where free-free emission dominates are identified by the frequency dependence $\sim v^{-2}$ in the optically thin part, and in those regions, the electron density along with an upper limit on the magnetic field can be derived. This approach was repeated by Komm et al. (1997) and Bong et al. (2003) for studies of other active regions. The advantage of this approach is obviously that the information of the spatially resolved spectrum at each pixel is self contained. Thus, we can determine the dominant radiation mechanism and temperature from the spectrum directly, and the turnover frequency gives information on the magnetic field at the pixel location with no need to guess. This diagnostic becomes more powerful with an increasing number of observing frequencies, which provides the main motivation for building a frequency-agile imaging telescope dedicated to solar observations (see Sect. 5).

\subsection{Center-to-Limb Variation}

A long-term investigation of a solar active region has to deal with the change in the aspect angle due to solar rotation, and such observations of an active region during its passage from the center to limb can, in fact, serve as a stereoscopic observation. A stereoscopic observation can be used to study the angle-dependent properties of the gyro-resonant emission (see (9)). Lee et al. (1993b) presented the spectrum of emission from an active region during its passage and interpreted the center-to-limb variation as due to the increase of the effective harmonic at greater viewing angles, by comparison with model predictions (Zlotnik 1968b). Another use is a tomographic application, in which the altitude of the radio source is inferred from the images of the source obtained at multiple perspective angles. Lack of source height information is a common shortcoming to all astronomical diagnostics, and is particularly needed for using the uniqueness of gyro-resonant radiation that is emitted from a very thin layer. Aschwanden and Bastian (1994) implemented a solar-rotation-based stereoscopic method to estimate source heights under the assumption that the active region did not evolve much during rotation. In addition, they also aimed to determine the relative motion of radio sources with respect to the photospheric differential rotation. Using solar radio maps at a wavelength of $20 \mathrm{~cm}$ and stereoscopic correlations between radio maps obtained on six different days they established the presence of 66 radio source components associated with 22 active regions, including the lifetime, source motion relative to the standard differential rotation rate, and limb darkening. Among their results we cite the finding that the average altitude of $20 \mathrm{~cm}$ sources is $25 \pm 15 \mathrm{Mm}$ with $90 \%$ of the sources found at heights less than $40 \mathrm{Mm}$. The average diameter of discrete source structures is $48 \pm 15 \mathrm{Mm}$, implying a vertical/horizontal aspect ratio of approximately 0.5 . The long-lived sources ( $\gtrsim 5$ days) show a systematic variation of their altitude as function of the center-limb distance. This limb darkening was interpreted as an effect of angle dependent opacity.

\subsection{Inhomogeneous Temperature Structure}

The temperature diagnostic capability of radio emission has been relatively less emphasized compared with magnetic field diagnostics. In Sect. 3.2, for instance, we discussed the use of the frequency-dependent morphology only for outlining the area filled with magnetic fields above a certain field strength. The brightness distribution inside the boundary is also important as indicating the temperature distribution on the isogauss surface corresponding to the observing frequency. Furthermore, its frequency-dependent variation can be associated with the change of temperature distribution on consecutive isogauss surfaces. As another way to view this result, the imaging spectroscopy given in Sect. 3.3 shows the optically thick 
brightness temperature in each spatial pixel shows a little variation with frequency. This variation is not necessarily due to detection noise, but to the inhomogeneous distribution of electron temperature along the line of sight. Thus, radio brightness maps can reveal the temperature inhomogeneity along both the line of sight and the direction nearly orthogonal to it, which is useful in deriving the 3D temperature structure.

Another interesting and related phenomenon is $\mathrm{O}$ mode polarization found at the center of active regions. Both $\mathrm{X}$ and $\mathrm{O}$ mode opacities become greatly reduced at small viewing angles, $\theta \approx 0^{\circ}$, but due to subtle difference in their dependence on the angle and harmonic, we may view a deeper layer in one mode than in the other mode. The apparent $\mathrm{O}$ mode polarization results when the $\mathrm{O}$ mode source layer has actually higher electron temperature than in the X mode layer, which thus indicates temperature inhomogeneity in the center of umbra (Vourlidas et al. 1997; see also Lee et al. 1993a).

\subsection{Comparison with EUV and Soft X-ray Observations}

When information on coronal density and temperature are available at other wavelengths such as EUV and soft X-rays, we can exploit it either as a cross check of those derived from radio data or to assist interpretation of radio data (Schmahl et al. 1982; Strong et al. 1984; Webb et al. 1987). This task was systematically carried out during a series of studies called the Coronal Magnetic Structures Observing Campaign (CoMStOC) which was designed to make simultaneous observations of soft X-ray, radio, and photospheric magnetic fields (Schmelz and Holman 1991). The plasma electron temperature and emission measures determined from the X-ray data are used to predict the free-free emission expected at the observing frequencies, and these predictions are compared with the radio observations to derive information on the inhomogeneous distribution of coronal conditions above active regions. One of the major findings of CoMStOC is the presence of cool material, when the predicted $20 \mathrm{~cm}$ brightness temperatures are found to be higher than those observed (Schmelz et al. 1994; see also Webb et al. 1987). Another important result was determination of the magnetic field.

Figure 4 shows a more recent result of coordinated EUV and radio observations to determine the coronal magnetic field. In this work, Brosius et al. (2002) used the EUV spectra and images of NOAA 8108 obtained with the Coronal Diagnostic Spectrometer (CDS) and the Extreme-ultraviolet Imaging Telescope (EIT) aboard the SOHO satellite to derive the differential emission measure (DEM) and the plasma electron density for each spatial pixel (along each line of sight) within both regions. The radio observations were used to constrain the magnetic scale height and the gross temperature structure of the atmosphere. These, along with the DEM, electron density, and observed radio brightness temperature maps, were then used to derive the temperature distribution of the coronal magnetic field strength $B(T)$ that reproduced simultaneously the observed RCP and LCP emissions at the observing frequencies for each spatial pixel in the images. Magnetic field strengths corresponding to third harmonic gyro-emission at $4.9 \mathrm{GHz}$ (580 Gauss) are found in coronal plasmas at temperatures as high as $3.2 \mathrm{MK}$, while magnetic field strengths corresponding to third harmonic gyro-emission at $15 \mathrm{GHz}$ (1800 Gauss) are found in coronal plasmas at temperatures as high as 1.6 MK. $B(T)$ was then converted to $B(h)$ and compared with extrapolations from photospheric magnetograms to tell whether or not they are in the potential state (see also Brosius et al. 1997; Brosius 2004).

\subsection{Free-Free Emission as Density and Abundance Diagnostics}

The ubiquitous free-free emission will dominate the active region radio emission in regions where magnetic field is not strong enough for optically thick gyro-resonant emission. The 

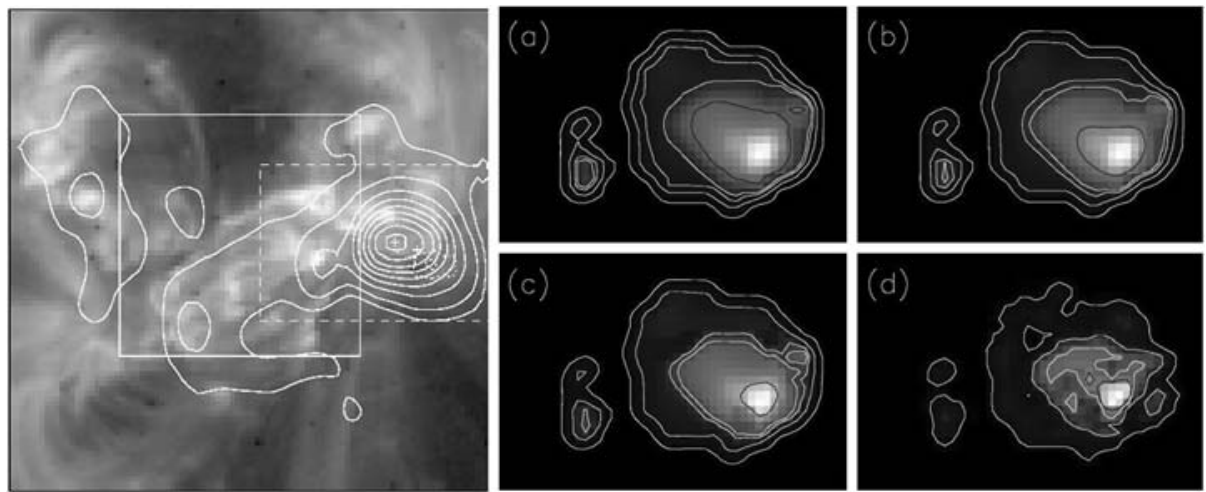

Fig. 4 Left: an EIT $195 \AA$ Amage of AR 8108 on 1997 November 18 . The whole field of view is $4^{\prime} \times 4^{\prime}$ and the solid contours correspond to radio brightness temperatures of $0.1,0.25,0.5,0.75,1.0,1.25,1.5,1.75$, and $2.0 \times 10^{6} \mathrm{~K}$ in the RCP at $4.866 \mathrm{GHz}$, measured with the VLA. The dashed box of dimension $1.9^{\prime} \times 1.3^{\prime}$ is the area chosen for analysis. Right: the resulting coronal magnetic strengths are shown at heights a 5000, b 10,000 , c 15,000 and $\mathbf{d} 25,000 \mathrm{~km}$ above the photosphere. Contour levels are 200, 579, 868, 1005 and 1508 Gauss where the last four values correspond, respectively, to $s=3,2$ at $4.866 \mathrm{GHz}$ and $s=3,2$ at $8.450 \mathrm{GHz}$ (from Brosius et al. 2002)

free-free emission is most sensitive to density and can typically be used to derive the electron density or emission measure $n_{\mathrm{e}}^{2} L_{\rho}$. To apply this density diagnostic we, however, need to confirm the dominance of the free-free emission in the region of interest and also know the turn-over frequency, $v_{\tau=1}(11)$. The temperature can be known from the optically thick spectrum. The imaging spectroscopy described in Sect. 3.3 is thus ideal for using free-free emission as a density diagnostic.

As an extension of this density diagnostic of free-free emission, White et al. (2000) presented a method for determining the abundance of Fe relative to $\mathrm{H}$ using radio and EUV data. Figure 5 shows the radio map of free-free emission compared with coordinated CDS and EIT images. One might think that EUV and radio images look quite different. However, for the compact, cool active region-which was identified with a free-free emission source-all these emissions show a morphological similarity to each other at the similar resolution. This confirms that all these radiations are commonly sensitive to density, but we know that the radio free-free emission is entirely due to electron density and EUV Fe line emission involves the abundance of $\mathrm{Fe}$. The ratio between the radio flux and EUV flux then gives rise to a measurement of $\mathrm{Fe} / \mathrm{H}$ in the solar corona: they found $\mathrm{Fe} / \mathrm{H}=1.56 \times 10^{-4}$, about four times higher than in the solar photosphere. This implies that low first ionization potential (FIP) elements such as Fe are enhanced in the solar corona relative to photospheric values (see, for reference of FIP, Laming 2004; Feldman and Widing 2003; Feldman et al. 1992).

\subsection{Free-Free Emission for Magnetic Field}

The polarization of free-free emissions has been observed with the RATAN-600 (Bogod and Gelfreikh 1980), the VLA (Schmelz et al. 1992) and the NoRH (Shibasaki et al. 1994; Gelfreikh and Shibasaki 1999). If the observation is made at a frequency where both RCP and LCP emissions are optically thin, one can simply determine the line-of-sight magnetic field in the source from the degree of circular polarization (12) assuming that the 


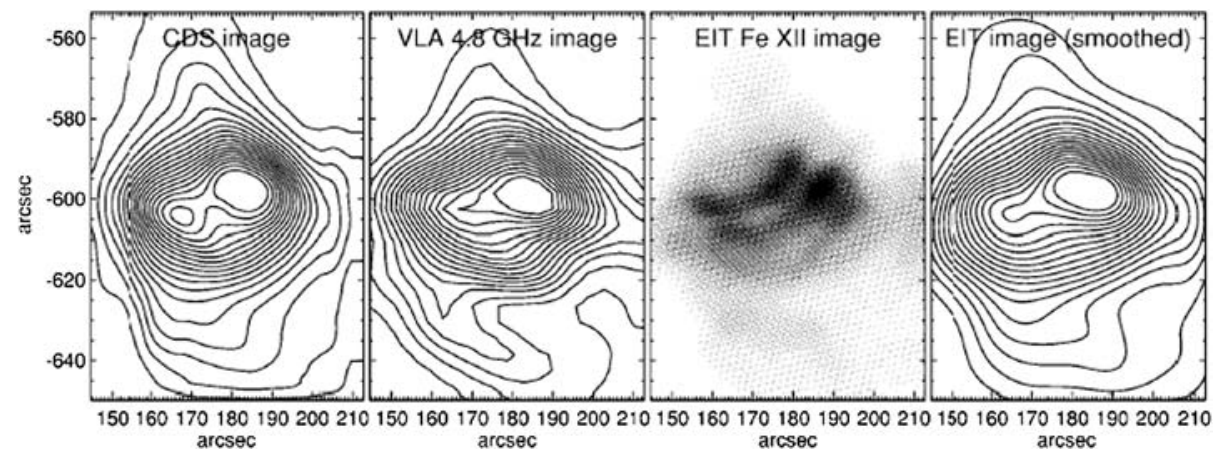

Fig. 5 Images of AR 8105 obtained by CDS (left panel: EUV image smoothed with a 7" Gaussian), VLA (second panel: $4.8 \mathrm{GHz}$, beam size 13" ), EIT FeXII $195 \AA$ A (third panel: gray scale, $5.2^{\prime \prime}$ resolution), and the EIT image smoothed to $10^{\prime \prime}$ resolution and contoured for direct comparison with the CDS and VLA images. In the contour images, contours are plotted at $5 \%, 10 \%, \ldots, 95 \%$ of the maximum for that image (from White et al. 2000)

source is homogeneous. There can, however, be many alternative cases where such a condition is not met. Gelfreikh (2004) presented a comprehensive technique that can deal with these variations: either both modes are optically thick or only one mode is thick while the other is thin in inhomogeneous sources. Gelfreikh (2004) included the contribution from the chromosphere as well. The result demonstrates that polarized free-free emission can be used as a temperature and magnetic field diagnostic almost as effectively as gyro-resonant radiation if the instrumental sensitivity is sufficient. Brosius et al. (1993) derived coronal magnetograms from free-free radio emission based on VLA and SERTS sounding rocket observations. As expected, free-free diagnostics are important in the places where the field strength is insufficient for gyro-resonance at low harmonics in active regions or in weaker field regions such as prominences and coronal holes (Gelfreikh 2004) as well as network fields (Chiuderi-Drago et al. 1983, 1987; Gary et al. 1990; Keller and Krucker 2004).

\subsection{Depolarization due to Mode Coupling}

We can generally expect the observed circular polarization maps to coincide with the photospheric magnetic polarity distribution in the way that the RCP (LCP) emission appears above the positive (negative) magnetic polarity regions, because the radio emission layers are fairly close to the photosphere. This indeed works well for isolated unipolar regions. For complex and extended active regions, the observed sense of circular polarization may sometimes disagree with the photospheric magnetic polarity distribution. The interpretation of such observations requires mode coupling theory (Sect. 2.3). In Fig. 6 we can see such a phenomenon by comparing the maps of circular polarization (lower row) with the optical active region (upper row). The polarization maps at two well-separated frequencies show the obvious trend that the zero polarization lines (where $V_{\mathrm{obs}}=0$ ) marked as $\mathrm{C}$ and $\mathrm{U}$, that is, the border between white $\left(V_{\mathrm{obs}}>0, \mathrm{RCP}\right)$ and black $\left(V_{\mathrm{obs}}<0, \mathrm{LCP}\right)$ colored regions moves away from the magnetic neutral line (NL) in the photosphere progressively with increasing frequency. This zero polarization line is called the depolarization strip, because the radiation from the region was probably intrinsically polarized but is unpolarized after propagating through the strip. The originally unpolarized region $\left(V_{\text {int }}=0\right)$ must be lying close to the photospheric NL, which is now polarized. This is why the zero polarization lines, $\mathrm{C}$ 


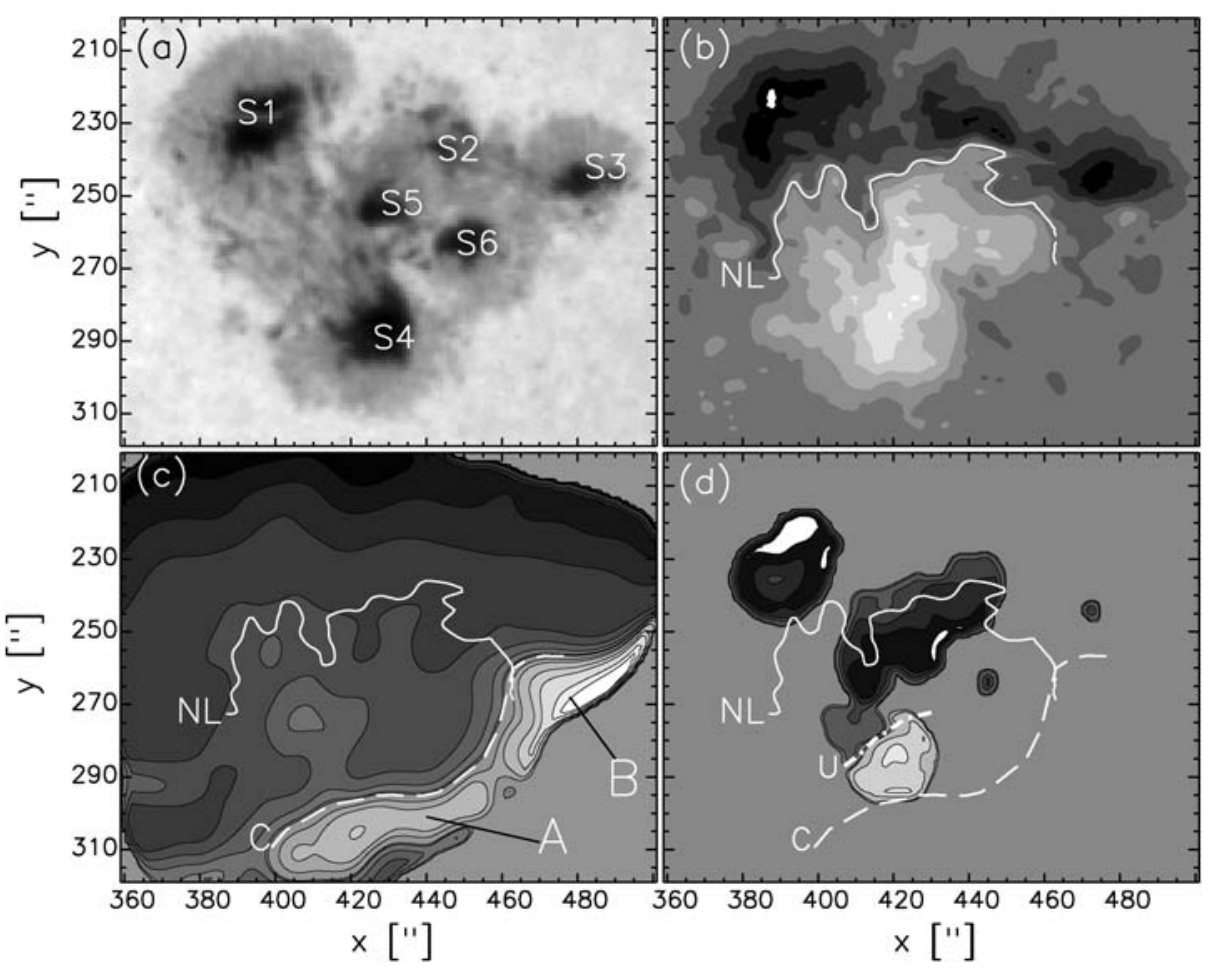

Fig. 6 Radio maps of the degree of circular polarization taken with the VLA at $4.9 \mathrm{GHz}$ and $15 \mathrm{GHz}$ (lower row) are shown in comparison with a white-light picture and a longitudinal magnetogram (upper row) of a complex active region, AR 6615. At both frequencies the sense of circular polarization does not correspond to magnetic polarity in the region above the sunspot $\mathrm{S} 5$. The depolarization lines, $V=0$, at $4.9 \mathrm{GHz}$ (dashed line $C$ ) and at $15 \mathrm{GHz}$ (dot-dashed line $U$ ) are noticeably shifted from the NL of longitudinal photospheric magnetic fields (solid line). The $(x, y)$ coordinates are geocentric coordinates in the west and the south, respectively, and $x$ and $y$ increase toward the west and the south, respectively (from Lee et al. 1998b)

and U, lie away from the photospheric NL. Since depolarization can occur only at the QT layer, the southward shift of the strip with frequency indicates that the magnetic polarity distribution changes with height in such a way that the negative polarity region expands to the south.

In depolarization strips, we can apply the condition $Q=\pi / 2 \ln 2$ in order to determine either the magnetic field or the density, typically by assuming one to allow the other to be derived. Kundu and Alissandrakis (1984) exploited this diagnostic using $5 \mathrm{GHz}$ polarization observations made with the WSRT on several consecutive days to determine the field strength under the assumed electron density of $\sim 10^{8} \mathrm{~cm}^{-3}$. Alissandrakis et al. (1996), on the other hand, used a linear force-free field model to determine the height and magnetic field and then derived a density in the corona. Lee et al. (1998b) used the two frequency polarization maps shown in Fig. 6 to determine densities at two different coronal heights with magnetic field models (the potential and the nonlinear force-free field extrapolations) available for locating the QT layer so that the magnetic field models could be tested based on the derived density.

While this diagnostic uses only the complete depolarization, $V_{\mathrm{obs}}=0$, it is, in principle, available for partial depolarization, that is, $0<\left|V_{\text {obs }} / V_{\text {int }}\right| \leq 1$ as well. In fact, one can 
rewrite (13) to express the magnetic field as a function of $V_{\mathrm{obs}} / V_{\text {int }}$. The latter can be used only when we have information on $V_{\text {int }}$ at a given time. Ryabov et al. $(1999,2005)$ constructed a map of $V_{\text {obs }} / V_{\text {int }}$ from radio observations with the NoRH and the SSRT where they adapted the previous day polarization map observed before the mode coupling occurs for the original polarization $V_{\text {int }}$ assuming no time-dependent change. These relative polarization maps were then converted into the distribution of magnetic field over a finite area over the QT layer in the high corona.

\subsection{Coronal Oscillations}

Oscillations are an omnipresent phenomenon on the solar surface. Whereas the 5-min oscillations are found in the photosphere and widely used for solar seismology, 3-min oscillations occur in umbral chromosphere (see, for a recent review, Staude 1999). At radio wavelengths, some periodic motions were detected above stable gyro-resonant sunspot-associated sources and considered as oscillations in the low corona or transition region (TR). Gelfreikh et al. (1999) used $17 \mathrm{GHz}$ NoRH data to measure the circular polarization flux of several such sources as a function of time and found nearly harmonic oscillations with periods mostly between 120-220 s. The periodic fluctuations of the polarization flux were interpreted in terms of magnetohydrodynamic oscillations which result in variations of the size of the emitting gyro-resonant layer and its temperature. Shibasaki (2001) detected 3-min oscillations in the $17 \mathrm{GHz}$ emission of a sunspot for which he applied the values of density and temperature fluctuations deduced from Solar Ultraviolet Measurements of Emitted Radiation (SUMER) onboard SOHO to find a good agreement with the detected radio oscillation and attribute the 3-min oscillation to the resonant excitation of the cut-off frequency mode of the temperature plateau around the temperature minimum. Nindos et al. (2002) used VLA observations to detect spatially resolved oscillations in the total intensity and circular polarization emission of a sunspot-associated gyro-resonant source and found that the oscillations are intermittent in time and patchy in space. They interpreted the radio oscillations as due to variations of the location of gyro-resonant surfaces with respect to the base of the chromosphere-corona transition region, that is, either the magnetic field strength or/and the height of the base of the TR oscillates.

\subsection{Other Targets}

While radio observations of solar active regions have primarily focused on their stationary or instantaneous structures, time-dependent radio emissions have also been exploited for some topics. Gopalswamy et al. (1994b) observed transient brightenings at 2-cm wavelength using the VLA and found that the sources were located near a sunspot umbra, perhaps at the footpoints of an X-ray transient loop observed with Yohkoh, and may thus be associated with small-scale heating. Zhang et al. (1998) further studied the spatial structure of the transient radio brightenings in solar active regions using the VLA at multiple wavelengths. The long-term (10 days) evolution of an active region at radio wavelengths was studied by Lara et al. (1998). They observed changes of the physical conditions of the active region corona associated with the evolution of the photospheric magnetic field. Simultaneous brightenings in transition region lines and in radio emission of a quiet region were observed with the VLA and discussed in relation to the coronal heating in quiet regions (Krucker and Benz 2000; Krucker et al. 1997; Benz et al. 1997).

The radio properties of solar magnetic structures other than active regions have also occasionally been studied. Zirin et al. (1991) measured the radio brightness temperature 
spectrum of the quiet sun with OVSA during the 1986-1987 sunspot minimum, and fit the spectrum with an optically thin coronal source and an optically thick chromospheric source at $\sim 11,000 \mathrm{~K}$. Gopalswamy et al. (1991) carried out a survey of large-scale coronal structures including active regions, plages, filaments, and coronal holes using the VLA at 1.5 $\mathrm{GHz}$ over a one-week period to determine statistical properties of these magnetic structures. Their findings include the radio depression over filaments by a factor of 2 compared with the quiet sun and the change of radio polarization associated with an emerging flux region (EFR).

\section{The Science of Coronal Active Regions}

The radiative properties of the active region radio emissions are closely tied to the major scientific issues of active regions, the magnetic field, mainly because solar coronal active regions are magnetically dominated plasma, and the radio emissions are generated and propagating in interaction with ambient magnetic field in the corona. In this section we discuss selective scientific issues that can be addressed by radio observations, and discuss not only the presently known solutions but potential capabilities.

\subsection{Coronal Field Strength}

The ability to determine the coronal field strength is one major advantage of solar radio observations, and its importance should be obvious in view of the fact that the conventional magnetogram technique is playing a central role in solar physics with measurements of the photospheric magnetic field. There is, however, a subtle difference between coronal and photospheric magnetic fields. The photosphere is a high $\beta\left(\equiv 8 \pi p / B^{2}\right.$ where $p$ is pressure) plasma and the concentration of magnetic field in small discrete areas with field strengths as high as 1-2 kilogauss poses an important problem in magnetohydrodynamics of flux tubes. On the other hand, reaching the corona, the field lines may be fully relaxed to permeate the low $\beta$ plasma more or less uniformly, and a discrete magnetic field distribution may be smeared out, in which case magnetic topology becomes more important (Longcope 1996).

Here, we want to make the case in a situation where the connectivity is obvious (e.g., unipolar isolated sunspot). In that case the coronal and photospheric field strengths, $B_{\mathrm{c}}$ and $B_{\mathrm{p}}$, respectively, can work altogether to tell us how the coronal region maps into the photosphere under the magnetic flux conservation law

$$
B_{\mathrm{p}} l_{x} l_{y}=B_{\mathrm{c}} L_{x} L_{y},
$$

where $l_{x} l_{y}$ is the rectangular area of the magnetic flux bundle in the photosphere and $L_{x} L_{y}$ is the corresponding area in the corona. Since the left-hand side can be obtained from a conventional magnetogram, the knowledge of $B_{\mathrm{c}}$ provided from radio data will allow us to determine the ratio of $L_{x} L_{y}$ to $l_{x} l_{y}$. This ratio can tell us which physics determine the extent of the magnetic field expansion in the corona, for instance, whether it simply diverges as in a vacuum or is confined by a pressure gradient or some self-confinement mechanism.

There are many radio observations indicative of strong fields in the corona. An NoRH observation of particularly large sunspots found field strengths of $B_{\mathrm{c}}=2000$ Gauss or more in the corona (Shibasaki et al. 1994). In VLA observations, the coronal fields of 1800 Gauss are found to be present in the corona of an active region with the maximum line-of-sight field in the photosphere not much more than 2000 Gauss (White 2004; 
White et al. 1991). Brosius and White (2006) used EUV and radio observations to measure coronal magnetic field strengths of 960 Gauss above a large sunspot at the limb. These results imply that strong active regions have so much flux that there is little field-free volume to expand into, and so field strength declines much less rapidly with height than simple models tend to predict (Akhmedov et al. 1982). The results for the strong magnetic fields in the corona are consistent with the recent coronal line measurements above active regions at the limb with an infrared polarimeter (Lin et al. 2000). This also agrees with the finding from loop width measurements that coronal loops tend to have constant widths, rather than showing expansion at greater heights (McClymont and Mikić 1994; Klimchuk 2000). Although the radio observations may not provide enough spatial resolution to resolve the flux tube expansion geometry, radio techniques are not dependent on unknown filling factors and can provide such geometrical information under the magnetic flux conservation principle as described earlier.

\subsection{Magnetostatics}

Active regions are, except during flares, only slowly changing over time, from tens of hours to days, and their magnetic configurations become the domain of magnetostatics. The relevant force-balance equation for solar active regions is (Low 1990)

$$
\frac{1}{4 \pi}(\nabla \times B) \times B-\nabla p-\rho g \hat{z}=0,
$$

where $g$ is gravity. These terms represent the Lorentz force, pressure gradient, and the gravity, respectively. There is as yet no way to completely determine the current density, $\nabla \times B$, directly from any observations, as it requires three orthogonal vector components at two spatial points along each axis. As an approximation, however, we can make use of the simple analytical solutions under symmetry (Schluter and Temesvary 1958; Low 1980) that have been found to be adequate for round sunspots. Lee et al. (1993c) derived both the vertical and radial distribution of the magnetic field of an isolated spot using multifrequency data from OVSA. Other studies also measured the pressure difference required to make the active region be as confined as observed (Nindos et al. 1996; Vourlidas et al. 1997).

A more simplified approach, but capable of accommodating complex active regions, is to consider the magnetostatics without the pressure and gravity terms as they are ignorable anyway in a low $\beta$ plasma. In this case, we end up with a single term describing the forcefree field

$$
\nabla \times B=\alpha B
$$

In solar physics, solving this equation using the observed photospheric magnetogram as a boundary condition is known as a coronal field extrapolation. This technique is classified according to the assumption on $\alpha$ : the potential field $(\alpha=0)$ ), linear force-free field (constant $\alpha$ ) and non-linear force-free field (spatially varying $\alpha$ ) extrapolations (Nakagawa and Raadu 1972; Sakurai 1979, 1982; Alissandrakis 1981; Gary 1989; Jiao et al. 1997; Amari et al. 1999). After Alissandrakis et al. (1980) used the potential field model for interpretation of their radio maps, it has been a common practice for solar radio astronomers to fit the radio data to theoretical radio emissions calculated using one of these magnetic field models. Lang et al. (1993) and Vourlidas (1996) used the potential field extrapolation to model thermal structure of active regions. Alternatively, direct comparisons between the observed coronal field implied by the radio images and the potential field model were also made to locate 
the presence of coronal currents, using polarized free-free emission (Schmelz et al. 1992) and gyro-resonant source areas (Lee et al. 1997). In any case, the radio data are not directly proving $\nabla \times B \neq 0$ but rather indirectly argue for the presence of currents based on a field strength comparison, that is, $|B|>\left|B_{\mathrm{p}}\right|$. Since the magnetic field strength varies rapidly with height, the height of the radio source becomes a critical factor in this argument. Once the height is known to a good precision, such a field strength comparison may allow us not only to locate the coronal current, but to determine its magnitude. Typically one has to adjust the assumption on $\alpha(r)$ until an agreement between the radio map and the magnetic field model is obtained (Lee et al. 1997).

More recently, a state-of-art technique has developed to solve the full magnetohydrodynamic equations (i.e., equations for mass and energy conservation in addition to (16)) to model the equilibrium magnetic field, temperature and density in 3D with a specific coronal heating rate (Mok et al. 2005). Gary et al. (2007) computed theoretical radio emission maps and spectra expected from the model, and tested them for the radio diagnostic capability, to find an excellent agreement between the input model and the values derived from the simulated radio data when high spectral resolution is available.

\subsection{Magnetic Neutral Line in the High Corona}

An active region magnetic loop may be blown out under an instability to become a Coronal Mass Ejection (CME) moving outward from the Sun (Low 2001). When the CME reaches the Earth, it may cause a geomagnetic storm if its magnetic polarity is oriented southward so as to reconnect with the Earth's magnetic field (Dungey 1961; Wilcox et al. 1980; Suess et al. 1984). The magnetic polarity distribution in the outermost active region corona is therefore of new interest in relation to space weather (see, for a recent review, Gopalswamy 2006). One may use, as proxy, the NL measured in the photospheric magnetogram (Yurchyshyn et al. 2005). However, the magnetic NL will gradually change with height depending on how the magnetic flux in each polarity competes with that in the other polarity, and also whether strong current densities (given by $\nabla \times B$ ) exist in the corona. A better proxy can be the coronal NL predicted by either a potential or a force-free field extrapolation from the photospheric field measurement (Song et al. 2006). The force-free field extrapolations are, however, subject to several uncertainties including the $180^{\circ}$ ambiguity in the vector magnetic field measurement (Metcalf et al. 2006). The radio polarization map is a relevant quantity in this regard, as it can show the NL of coronal fields directly. With radio polarization maps at multifrequencies, we can even trace the change of NL orientation with height, as the depolarization strip appears close to the photospheric NL at higher frequencies (low corona) and moves gradually away from it at lower frequencies (high corona), revealing the location of the QT layer as a function of coronal height (see Sect. 3.9 and Fig. 6). Thus far the use of mode coupling has been focused on density or magnetic field diagnostics, without paying much attention to the location of the QT layer itself, despite its importance.

Besides, there are peculiar active region configurations that are known to be prone to magnetic eruptions. They include anemone active regions found in unipolar regions near coronal holes (Shibata et al. 1994) and sigmoidal active regions (Hudson et al. 1998) in bipolar configurations. Both features are discovered from soft X-ray images, and have received a great deal of attention in relation to space weather. A radio observation of the anemone structures has been presented by Vourlidas et al. (1996), with as yet no report on the radio sigmoids. Although many properties of these two types of active regions have been known from X-ray and magnetogram studies, the coronal magnetic polarity distribution of these active regions, as can be learned from radio polarization maps, may still shed new light on their magnetic field structure. 


\subsection{Sheet Current}

An important concept in theoretical MHD is the tangential discontinuity in a magnetic field which is a special place where magnetic fields with different topology may reconnect to each other. For the purpose of distinction, let us call this sheet current and the above-mentioned current distributed over a finite volume volume current. These two types of current differ from each other in many ways. From a physical perspective, it is likely that the sheet currents reside in very thin layers such as magnetic separatrices and lead to a rapid release of energy via magnetic reconnection (Longcope 1996), while the volume current is perhaps related to the steady coronal heating via topological dissipation (Parker 1988). From the diagnostic viewpoint, the volume current in the photosphere can be directly measured in the form of magnetic shear or twist appearing in a vector magnetogram, and in the corona that can be indirectly measured using the radio technique as described earlier. On the other hand, the sheet current is neither inferred from observations nor predicted by the force-free field extrapolations. Only recently the thin, bright features above post-CME loop systems detected by the Ultraviolet Coronagraph Spectrometer (UVCS) and Large Angle and Spectrometric Coronagraph Experiment (LASCO) have been claimed to be current sheets (Ko et al. 2003). The brightness in this case may imply energy dissipation in the current sheet to some extent, which in turn makes them observable. However, theoretically the current sheet may also exist without energy dissipation, in which case no direct observation will be available. Interestingly, the theoretical configuration of the sheet current exactly coincides with the configuration conceived as ideal for mode coupling (Zheleznyakov et al. 1996; Zheleznyakov and Zlotnik 1988). In both cases, the surrounding magnetic fields should reverse their orientation across the current sheet and the field-free region residing in the middle should be very thin (Harris 1962). Thus, the requirement for the tangential discontinuity is also an ideal condition for the strong coupling of polarized radiations passing through the current sheet. In this sense the radio depolarization phenomenon may become a unique tool for detecting magnetic tangential discontinuities in the corona, which are otherwise hardly visible. The possible detection of current sheets in radio polarization maps have been briefly discussed by Schmahl et al. (1984), Gopalswamy et al. (1994a), and Lee et al. (1998b).

\subsection{Coronal Seismology}

Coronal waves and oscillations recently became an important branch of solar magnetohydrodynamics with the unprecedented high resolution coronal observations at EUV wavelengths (Nakariakov and Verwichte 2005). In comparison with the traditional helioseismology, which involves the acoustic waves in global scale, the coronal seismology deals with various waves: Alfvén, magneto-acoustic, and sound waves in localized magnetized coronal regions. Obviously radio emission can join this growing area of solar physics with its sensitivity to the coronal magnetic field. As yet, only a few radio studies have dealt with the oscillatory phenomena in active region radio emissions (Sect. 3.10). A reason could be that radio observations lack sufficient spatial resolution to measure the oscillatory motions directly on images (as done with TRACE and EIT) and that no direct measurement of the Doppler shifts can be made (available from spectral line observations with CDS, SUMER, and Yohkoh/BCS). However, the radio observations can be made with higher time cadence and, more importantly, represent the oscillations in magnetic field (if detected from a gyroresonant source), unlike the intensity or velocity oscillations measured at other wavelengths. Nindos et al. (2002) carried out a model analysis of the spatially resolved oscillations of a sunspot gyro-resonant source to see whether they are due to oscillations of the magnetic 
field strength (magneto-acoustic waves) or magnetic field angle (Alfvén waves). They found that the radio oscillations due to directional oscillations of the magnetic field would be too small to be detectable with current instruments, and suggested that additional information from velocity oscillations is needed to identify the dominant wave mode. Instead of the Doppler shift that cannot be measured with radio continuum data, we expect that variations in the turnover frequency may be measured and used as an indicator of the magnetic field oscillation if sufficiently high-resolution spectroscopy is available in future. Once the origin and nature of the observed oscillations in radio emission are understood, we may use the radio oscillatory phenomena for coronal seismology.

\subsection{Characteristic Speeds}

The Alfvén and sound speeds are the fundamental velocity scales in a medium governed by MHD, and are given by

$$
V_{\mathrm{A}}=\frac{B}{\sqrt{4 \pi \rho}}, \quad C_{\mathrm{S}}=\sqrt{\frac{k_{\mathrm{B}} T_{\mathrm{e}}}{m_{\mathrm{e}}}} .
$$

The parameters involved with these speeds - that is, temperature, density, and magnetic field strength - can be derived from either gyro-resonant or free-free emissions. Specific physical processes of interest are the magnetic loop oscillation (see above) and shock formation. Typical values found from EUV images are $V_{\mathrm{A}} \sim 1000 \mathrm{~km} \mathrm{~s}^{-1}$ and $V_{\mathrm{s}} \sim 200 \mathrm{~km} \mathrm{~s}^{-1}$, and scales involved with oscillations are $10^{3}-10^{5} \mathrm{~km}$. However, Alfvén speeds as high as $20,000-60,000 \mathrm{~km} \mathrm{~s}^{-1}$ are also derived with coordinated EUV and radio observations (Brosius et al. 2002; Brosius and White 2006). Periodic motions from $1 \mathrm{~s}$ to several min would belong to the regime of MHD waves (Aschwanden et al. 1999; Nakariakov et al. 1999). Although the TRACE imaging directly reveals the coronal loop oscillation, the absolute scales of these natural speeds are still essential for quantitative analysis, for example, determination of harmonic and modes. In the radio community, the Alfvén speed was derived during the earlier mentioned CoMStOC (Sect. 3.6) campaign which was designed to determine both magnetic field and density together.

Another place where we need the Alfvén speed is in studying magnetic field reconnection. The central quantity in the theory is the velocity of the incoming magnetic field lines into the reconnection point normalized by the local Alfvén speed, that is, the Alfvén Mach number. This is defined as the magnetic reconnection rate, which is also equivalent to the aspect ratio of the reconnecting current sheet. Magnetic reconnection itself is beyond the scope of the active region study, but the background Alfvén speed can be determined by the active region radio emissions. In the well-known Kopp-Pneuman type magnetic reconnection (Kopp and Pneuman 1976), the magnetic flux invariant is used under the translational symmetry along a magnetic arcade. Setting the axis of the magnetic arcade to be the $y$-axis, that is, $L_{y}=l_{y}$, we have, from the time derivative of (14), the following relation (Priest and Forbes 2002)

$$
B_{\mathrm{p}} v_{\mathrm{p}}=B_{\mathrm{c}} V_{\mathrm{c}},
$$

where $v_{\mathrm{p}}=\mathrm{d} l_{x} / \mathrm{d} t$ and $V_{\mathrm{c}}=\mathrm{d} L_{x} / \mathrm{d} t$. It is now common that $v_{\mathrm{p}}$, the transverse velocity of a photospheric feature (flare ribbons) associated with the coronal reconnection point, is identified with the flare ribbon velocity. Knowledge of $B_{\mathrm{c}}$ will then allow us to determine $V_{\mathrm{c}}$, the incoming speed of magnetic field lines into the reconnection point. $V_{\mathrm{c}}$ itself is an important measure for magnetic reconnection, but theoreticians prefer it normalized by the local Alfvén speed, because $V_{x} / V_{\mathrm{A}}$ identifies the nature of the magnetic reconnection process, 


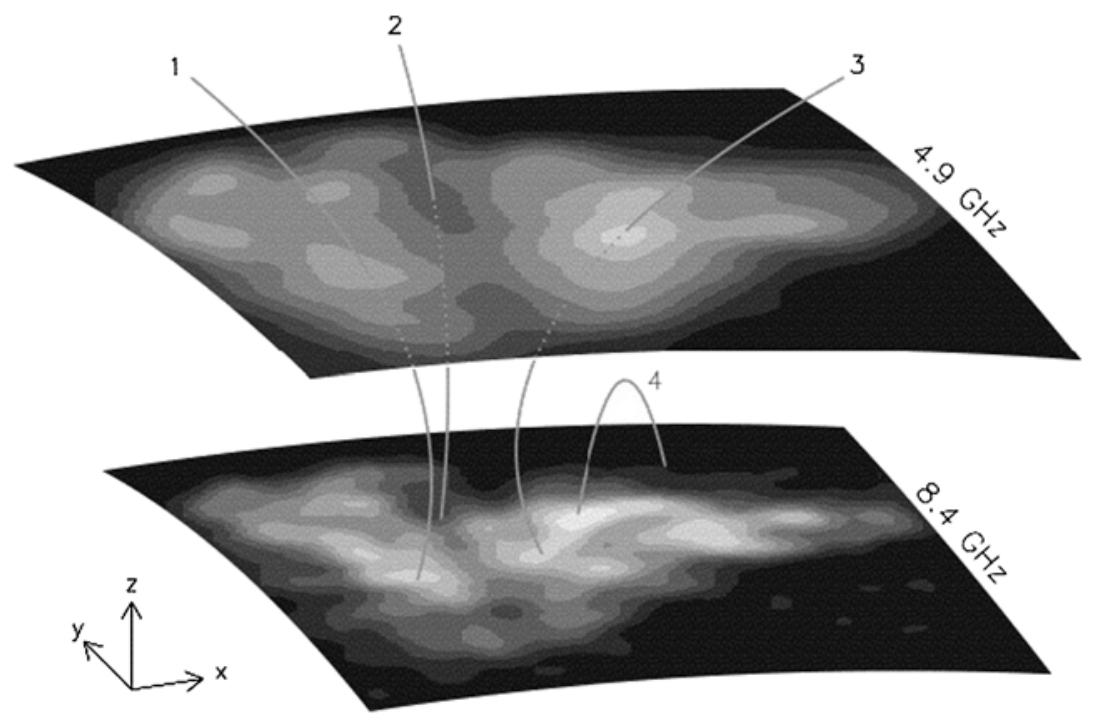

Fig. 7 Illustration of the proposed method. The two surfaces shown are (simplified) hypothetical emission layers for 4.9 and $8.4 \mathrm{GHz}$, respectively, and the gray-scale images on the surfaces are the actual observed $\mathrm{X}$-mode radio images of AR 6615. The coordinates $(x, y)$ form the sky plane, while $z$ is the line of sight. Several magnetic field lines connecting the two emission layers are shown. Magnetic field lines numbered 1-3 should connect regions of similar temperatures on the two layers if they are correctly predicted by the photospheric field extrapolation, but there can be field lines such as line 4 that do not pass both layers, but pass only one layer (from Lee et al. 1999a)

specifically, whether it is Sweet-Parker- or Petschek-type reconnection (see Aschwanden 2005, pp. 409-411). Therefore both magnetic field and density in the background should be determined from radio data to be used in the magnetic reconnection study.

\subsection{Active Region Heating and Magnetic Topology}

To understand coronal temperatures observed to be as high as several million degrees, we need the physics of coronal heating. Since radio brightness maps directly represent actual temperature distributions on a thin isogauss surface, it may be argued that radio data hold a major promise for resolving the coronal heating problem. Although radio observations can certainly reveal the absolute temperature of a given active region in a straightforward manner, the soft X-ray community has emphasized that the coronal temperatures are organized along field lines and the magnetic field connectivity is more important (Rosner et al. 1978). The importance of the magnetic connectivity in coronal temperature structure is expected, because heat transport parallel to the magnetic field is so much faster than it is across magnetic field lines.

This relationship between magnetic topology and temperature has rarely been used in radio studies, perhaps because observed radio maps are temperature distributions on isogauss surfaces rather than temperature variations along the magnetic loops. There are, however, several ways for using radio maps for temperature studies. For instance, one can combine the fact that radio maps at multifrequencies represent temperature on different isogauss surfaces with the idea that temperatures measured at two different locations on a field line should be very well correlated, as schematically shown in Fig. 7. Lee et al. (1999a) compared this correlation for the field lines from potential and force-free extrapolations using 
brightness temperatures measured at 5 and $8 \mathrm{GHz}$ and showed that indeed the correlation was extremely good for the nonlinear force-free extrapolation and much poorer for the other extrapolations. A more quantitative approach is to adopt several trial heating functions and solving a heat transfer equation to determine the temperature along a given field line. Lee et al. (1998a) applied several trial heating functions parameterized in terms of magnetic field, force-free parameter $\alpha$, and current density together with Rosner et al. (1978) scaling law to interpret high resolution radio maps of a complex active region shown in Fig. 2. The best agreement between the model and the observations was found when the heating function is proportional to the magnetic field strength at the footpoint, in favor of Parker's hypothesis of coronal heating by nanoflares (Parker 1988).

\section{Conclusions}

We have reviewed the theory behind, observations of, and scientific issues involved with solar active region radio emissions to demonstrate that gyro-resonant emission, free-free emission, and the mode coupling phenomenon offer excellent opportunities to explore coronal physics. To close, we briefly compare the radio diagnostics for coronal magnetic fields with those in other wavelengths, identify the unique aspect of active region radio emissions, and finally suggest future directions for solar radio emission study.

Many radio studies have mainly emphasized the radio diagnostic ability to determine coronal magnetic fields and implemented so-called coronal magnetography in four different contexts: (1) imaging spectroscopy (Gary and Hurford 1994), (2) coordinated EUV and radio observations (Brosius et al. 1993, 1997, 2002), (3) polarized free-free emission (Gelfreikh 2004), and (4) mode coupling phenomena (Ryabov et al. 1999, 2005; Ryabov 2004). On the other hand, the recent studies at EUV/UV and soft X-ray wavelengths also emphasized a branch of solar physics where magnetic topology plays an important role. Perhaps radio studies in future may need to pay more attention to those properties of radio emissions related to the topology issue so that they can better contribute to this recent trend in solar physics. We presented several ideas toward this goal: (1) The mode coupling phenomenon has mostly been used for density diagnostics, but can be used to identify the orientation of magnetic polarity. (2) The variation of brightness morphology with frequency can be used to derive the magnetic field strength distribution, but it can also be used to trace how temperature is organized in 3D. (3) The observed radio polarization may be used to locate the magnetic tangential discontinuity if mode coupling occurs strongly in current sheets. (4) The measurement of coronal field strength can, under the magnetic flux conservation law, be used to map a specific coronal volume back to its photospheric counterpart. In any case, these radio properties lead to inference of the magnetic topology, whereas the EUV and soft X-ray images show the topology more directly. It is therefore necessary for radio emission studies to make use of the EUV and soft X-ray images, whenever available, and present those not directly seen at each wavelength, for example, absolute values of magnetic field strength and temperature.

If this review has to select the most powerful aspect of active region radio emissions, it would be the fact that the radio emission is a continuum, and its sensitivity to the relevant parameters is uniform across a wide range of the parameters, as a monotonic function of frequency. The radio emission may thus be more suitable for studying the corona as a low $\beta$ plasma. This property is not shared by spectral line radiations at EUV and soft X-ray wavelengths that are sensitive to a narrow range of parameters, which has its own advantage in revealing the magnetic topology. The unique frequency dependence of radio emission is 


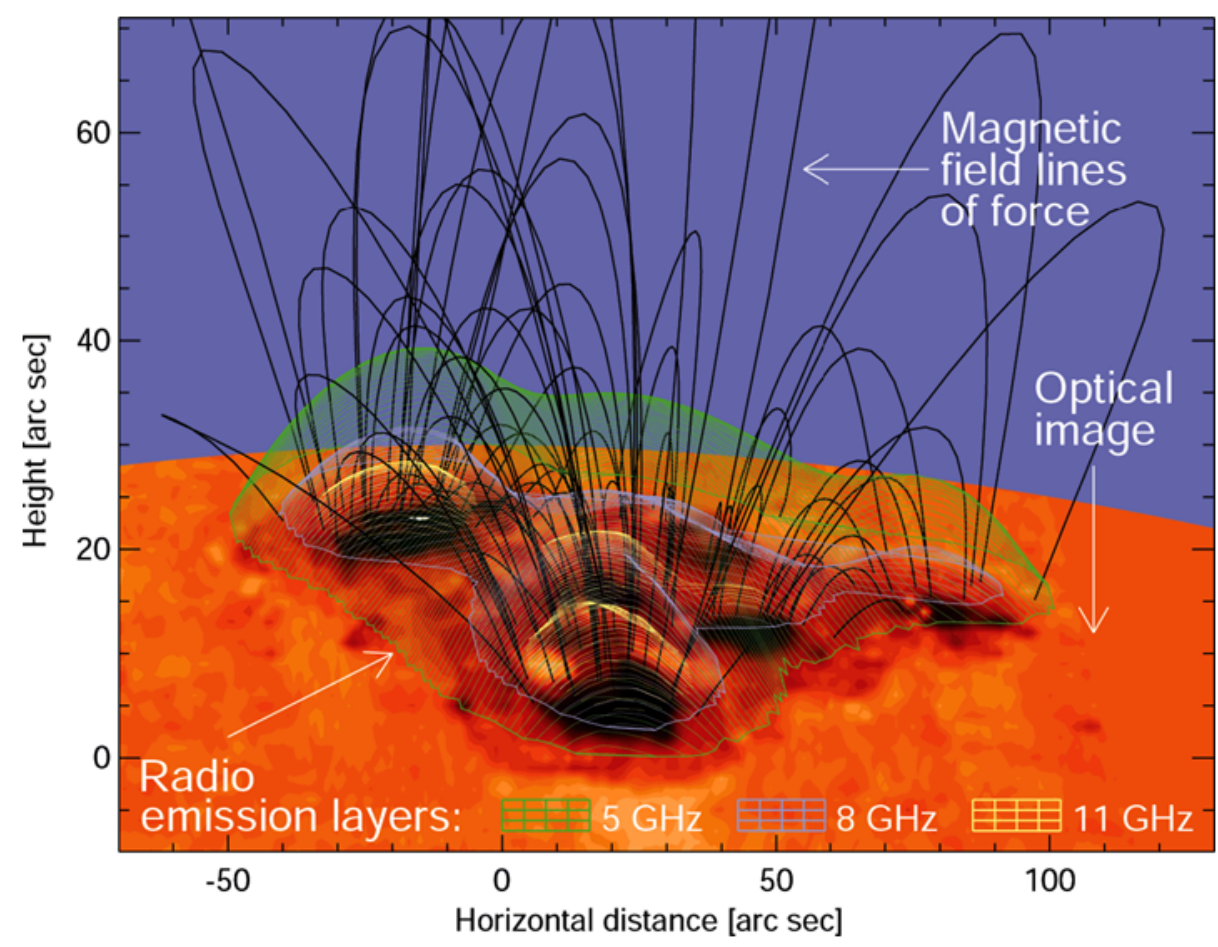

Fig. 8 Radio emission layers versus magnetic field line topology. A perspective view of a complex sunspot group (AR 6615 on 1991 May 7) in optical continuum is shown with field lines extrapolated into the corona using a nonlinear force-free extrapolation by Z. Mikic (see Lee et al. 1998a). The three meshed surfaces are the calculated gyroresonant surfaces in the corona that will dominate the radio opacity at each of three radio frequencies: $5 \mathrm{GHz}$ ( $B=600$ Gauss), $8 \mathrm{GHz}$ ( $B=950$ Gauss) and $11 \mathrm{GHz}(B=1300 \mathrm{Gauss})$

also important in resolving the common problem in all astronomical imaging that the source height along the line of sight cannot be determined. Although radio observations share this shortcoming, the systematic change of morphology with frequency can, at least, suggest how to trace the structural variation of the source in 3D. To illustrate this idea, we refer to Fig. 8 and presume that the temperature on an isogauss surface would be seen in a single frequency radio map while some bright field lines appear in the relative brightness distribution at EUV and soft X-rays. It may be unclear how to correlate them to each other since they give different information in rather orthogonal directions. However, multi-frequency radio maps provide multiple slices of the corona that progressively scan the corona along the direction similar to the field lines, and thus could complement the measurement of topology alone. This diagnostic power increases in proportion to the number of observing frequencies and yields stronger constraints on the field line related issues.

Currently solar radio astronomers are constructing a more powerful solar telescope called the Frequency Agile Solar Radio telescope (FASR) that will deliver to the solar community a higher level of imaging spectroscopy (Bastian 2004). Along with the new hardware, software improvement is needed in order to improve the image quality. In addition to the standard CLEAN imaging technique, both self-calibration (White et al. 1992) and maximum entropy method (Nindos et al. 1999) are found to work well for solar imaging. To accommodate the change of resolution of radio maps as a function of frequency, an essential aspect of imaging 
spectroscopy, an imaging technique called Spatio-Spectral Maximum Entropy Method (SSMEM) has been developed (Bong et al. 2005, 2006). With this instrument, we may expect further advances in the study of solar radio emissions as follows: (1) 3D active region structure can be better explored by fully exploiting the strength of radio diagnostics (discussed earlier), namely, the additional constraint imposed by the frequency dependent variation of radio maps. (2) The very interesting science associated with polarization, as emphasized in this paper, can be addressed with the improved sensitivity of the FASR. (3) The targets can include fainter radio sources such as plages, filaments, EFRs and coronal holes. (4) The target science can extend from the traditional study of stationary active region structures to other topics that require long-term monitoring of solar radio sources, including rapid transient brightenings and long-term variability of the radio emissions in active regions, coronal seismology, and solar cycle dependence of coronal structure. These topics have received less attention at radio wavelengths due to the limited observing times available for solar radio observations. With FASR's dedication to solar observations along with its enhanced ability for imaging spectroscopy, the physics underlying these radio emissions will, we hope, be addressed in the future.

Acknowledgements I thank Dr. Stephen M. White and an anonymous referee for their helpful comments. This work was supported by NSF grant AST 06-07544 and NASA grant NNG0-6GE76G.

\section{References}

S.B. Akhmedov, G.B. Gelfreikh, V.M. Bogod, A.N. Korzhavin, Sol. Phys. 79, 41 (1982)

C.E. Alissandrakis, Astron. Astrophys. 100, 197 (1981)

C.E. Alissandrakis, M.R. Kundu, Astrophys. J. (Lett.) 253, L49 (1982)

C.E. Alissandrakis, M.R. Kundu, Astron. Astrophys. 139, 271 (1984)

C.E. Alissandrakis, M.R. Kundu, P. Lantos, Astron. Astrophys. 82, 30 (1980)

C.E. Alissandrakis, F. Borgioli, F. Drago, F. Chiuderi, M. Hagyard, K. Shibasaki, Sol. Phys. 167, 167 (1996)

T. Amari, T.Z. Boulmezaoud, Z. Mikic, Astron. Astrophys. 350, 1051 (1999)

E.V. Appleton, J. Instrum. Electron. Eng. 71, 642 (1932)

M.J. Aschwanden, Physics of the Solar Corona. An Introduction with Problems and Solutions, 2nd edn. (Springer, New York Berlin, 2005)

M.J. Aschwanden, T.S. Bastian, Astrophys. J. 426, 425 (1994)

M.J. Aschwanden, L. Fletcher, C.J. Schrijver, D. Alexander, Astrophys. J. 520, 880 (1999)

M.J. Aschwanden, D. Alexander, M.L. Derosa, in Solar and Space Weather Radiophysics-Current Status and Future Developments (Kluwer, Dordrecht, 2004), Chap. 12

R. Bandiera, Astron. Astrophys. 112, 52 (1982)

T.S. Bastian, in Solar and Space Weather Radiophysics-Current Status and Future Developments (Kluwer, Dordrecht, 2004), Chap. 3

A.O. Benz, S. Krucker, L.W. Acton, T.S. Bastian, Astron. Astrophys. 320, 993 (1997)

V.M. Bogod, G.B. Gelfreikh, Sol. Phys. 67, 29 (1980)

S.-C. Bong, J. Lee, D.E. Gary, H.S. Yun, J. Korean Astron. Soc. 36, S29 (2003)

S.-C. Bong, J. Lee, D.E. Gary, H.S. Yun, J. Chae, J. Korean Astron. Soc. 38, 445 (2005)

S.-C. Bong, J. Lee, D.E. Gary, H.S. Yun, Astrophys. J. 636, 1159 (2006)

J.W. Brosius, in Solar and Space Weather Radiophysics-Current Status and Future Developments (Kluwer, Dordrecht, 2004), Chap. 13

J.W. Brosius, G.D. Holman, Astrophys. J. 342, 1172 (1989)

J.W. Brosius, S.M. White, Astrophys. J. 601, 546 (2004)

J.W. Brosius, S.M. White, Astrophys. J. 641, L69 (2006)

J.W. Brosius, J.M. Davila, W.T. Thompson, R.J. Thomas, G.D. Holman, N. Gopalswamy, S.M. White, M.R. Kundu, H.P. Jones, Astrophys. J. 411, 410 (1993)

J.W. Brosius, J.M. Davila, R.J. Thomas, S.M. White, Astrophys. J. 488, 488 (1997)

J.W. Brosius, E. Landi, J.W. Cook, J.S. Newmark, N. Gopalswamy, A. Lara, Astrophys. J. 574, 453 (2002)

F. Chiuderi-Drago, M. Felli, G. Tofani, Astron. Astrophys. 61, 79 (1977) 
F. Chiuderi-Drago, R. Bandiera, R. Falciani, E. Antonucci, K.R. Lang, R.F. Willson, K. Shibasaki, C. Slottje, Sol. Phys. 80, 71 (1982)

F. Chiuderi-Drago, M.R. Kundu, E.J. Schmahl, Sol. Phys. 85, 237 (1983)

F. Chiuderi-Drago, C.E. Alissandrakis, M. Hagyard, Sol. Phys. 112, 89 (1987)

M.H. Cohen, Astrophys. J. 131, 664 (1960)

A.E. Covington, Nature 159, 405 (1947)

A.E. Covington, Proc. IRE 37, 407 (1949)

V. Domingo, B. Fleck, A.I. Poland, Space Sci. Rev. 72, 81 (1995)

G.A. Dulk, D.E. Gary, Astron. Astrophys. 124, 103 (1983)

G.A. Dulk, Ann. Rev. Astron. Astrophys. 23, 169 (1985)

J.W. Dungey, Phys. Res. Lett. 6, 47 (1961)

U. Feldman, K.G. Widing, Space Sci. Rev. 107, 665 (2003)

U. Feldman, P. Mandelbaum, J.F. Seely, G.A. Doschek, H. Gursky, Astrophys. J. Suppl. 81, 387 (1992)

P.V. Foukal, Astrophys. J. 210, 575 (1976)

G.A. Gary, Astrophys. J. Suppl. 69, 323 (1989)

D.E. Gary, G.J. Hurford, Astrophys. J. 420, 903 (1994)

D.E. Gary, G.J. Hurford, in Solar and Space Weather Radiophysics-Current Status and Future Developments (Kluwer, Dordrecht, 2004), Chap. 4

D.E. Gary, C.U. Keller, Solar and Space Weather Radiophysics-Current Status and Future Developments (Kluwer, Dordrecht, 2004)

D.E. Gary, H. Zirin, H. Wang, Astrophys. J. 355, 321 (1990)

D.E. Gary, J. Lee, G. Giodano, Y. Mok (2007, in preparation)

G.B. Gelfreikh, in Solar and Space Weather Radiophysics-Current Status and Future Developments (Kluwer, Dordrecht, 2004), Chap. 6

G.B. Gelfreikh, B.I. Lubyshev, Sov. Astron. 23, 316 (1979)

G.B. Gelfreikh, V.P. Nefedev, Sov. Astron. Lett. 1, 127 (1975)

G.B. Gelfreikh, K. Shibasaki, in Magnetic Fields and Solar Processes, ed. by A. Wilson. The 9th European Meeting on Solar Physics, Florence, Italy, 12-18 September 1999. European Space Agency, ESA SP448 (1999), p. 197

G.B. Gelfreikh, V. Grechnev, T. Kosugi, K. Shibasaki, Sol. Phys. 185, 177 (1999)

N. Gopalswamy, Space Sci. Rev. 124, 145 (2006)

N. Gopalswamy, S.M. White, M.R. Kundu, Astrophys. J. 379, 366 (1991)

N. Gopalswamy, V.V. Zheleznyakov, S.M. White, M.R. Kundu, Sol. Phys. 155, 339 (1994a)

N. Gopalswamy, T.E.W. Payne, E.J. Schmahl, M.R. Kundu, J.R. Lemen, K.T. Strong, R.C. Canfield, J. de La Beaujardiere, Astrophys. J. 437, 522 (1994b)

E.G. Harris, Nuovo Cim. 23, 117 (1962)

D.R. Hartree, Proc. Camb. Philos. Soc. 27, 143 (1931)

G.D. Holman, M.R. Kundu, Astrophys. J. 292, 291 (1985)

H.S. Hudson, J.R. Lemen, O.C.S. Cyr, A.C. Sterling, D.F. Webb, Geophys. Res. Lett. 25, 2481 (1998)

G.J. Hurford, in Solar Flares and Coronal Physics Using P/OF as a Research Tool, ed. by E. TandbergHanssen, R.M. Wilson, H.S. Hudson, NASA Conf. Pub., vol. 2421 (1986), p. 191

L. Jiao, A.N. McClymont, Z. Mikić, Sol. Phys. 174, 311 (1997)

T. Kakinuma, G. Swarup, Astrophys. J. 136, 975 (1962)

C.U. Keller, S. Krucker, in Solar and Space Weather Radiophysics-Current Status and Future Developments (Kluwer, Dordrecht, 2004), Chap. 14

J.A. Klimchuk, Sol. Phys. 193, 53 (2000)

Y.-K. Ko, J.C. Raymond, J. Lin, G. Lawrence, J. Li, A. Fludra, Astrophys. J. 594, 1068 (2003)

R.W. Komm, G.J. Hurford, D.E. Gary, Astron. Astrophys. Suppl. Ser. 122, 181 (1997)

R.A. Kopp, G.W. Pneuman, Sol. Phys. 50, 85 (1976)

S. Krucker, A.O. Benz, Sol. Phys. 191, 341 (2000)

S. Krucker, A.O. Benz, T.S. Bastian, L.W. Acton, Astrophys. J. 488, 499 (1997)

M.R. Kundu, Solar Radio Astronomy (Interscience, New York, 1965)

M.R. Kundu, C.E. Alissandrakis, Sol. Phys. 94, 249 (1984)

M.R. Kundu, C.E. Alissandrakis, J.D. Bregman, A.C. Hin, Astrophys. J. 213, 278 (1977)

J.M. Laming, Astrophys. J. 614, 1063 (2004)

K.R. Lang, R.F. Willson, Astrophys. J. Lett. 255, L111 (1982)

K.R. Lang, R.F. Willson, V. Gaizauskas, Astrophys. J. 267, 455 (1983)

K.R. Lang, R.F. Willson, J.N. Kile, J. Lemen, K.T. Strong, V.L. Bogod, G.B. Gelfreikh, B.I. Ryabov, S.R. Hafizov, V.E. Abramov, S.V. Svetkov, Astrophys. J. 419, 398 (1993)

P. Lantos, Sol. Phys. 22, 387 (1972) 
A. Lara, N. Gopalswamy, M.R. Kundu, R. Perez-Enriquez, H. Koshiishi, S. Enome, Sol. Phys. 178, 353 (1998)

J. Lee, G.J. Hurford, D.E. Gary, Sol. Phys. 144, 45 (1993a)

J. Lee, G.J. Hurford, D.E. Gary, Sol. Phys. 144, 349 (1993b)

J. Lee, D.E. Gary, G.J. Hurford, H. Zirin, in The Magnetic and Velocity Fields of Solar Active Regions, ed. by H. Zirin, G. Ai, H. Wang. ASP Conf. Ser., vol. 46: IAU Colloq., vol. 141 (1993c), p. 287

J. Lee, S.M. White, N. Gopalswamy, M.R. Kundu, Sol. Phys. 174, 175 (1997)

J. Lee, A.N. McClymont, Z. Mikić, S.M. White, M.R. Kundu, Astrophys. J. 501, 853 (1998a)

J. Lee, S.M. White, M.R. Kundu, Z. Mikic, A.N. McClymont, Sol. Phys. 180, 193 (1998b)

J. Lee, S.M. White, M.R. Kundu, Z. Mikić, A.N. McClymont, Astrophys. J. 510, 413 (1999a)

J. Lee, S.M. White, M.R. Kundu, Z. Mikic, in Proc. of the Nobeyama Symp., ed. by T.S. Bastian, N. Gopalswamy, K. Shibasaki. NRO Report No. 479 (1999b), p. 65

H. Lin, M.J. Penn, S. Tomczyk, Astrophys. J. Lett. 541, L83 (2000)

D.W. Longcope, Sol. Phys. 169, 91 (1996)

B.C. Low, Sol. Phys. 67, 57 (1980)

B.C. Low, Ann. Rev. Astron. Astrophys. 28, 491 (1990)

B.C. Low, J. Geophys. Res. 106, 25141 (2001)

A.N. McClymont, Z. Mikić, Astrophys. J. 422, 899 (1994)

D.B. Melrose, Plasma Astrophysics (Gordon and Breach, New York, 1980)

D.B. Melrose, in Solar Radiophysics, ed. by D.J. McLean, N.R. Labrum (Cambridge University Press, Cambridge New York, 1985), p. 237

D.B. Melrose, Sol. Phys. 43, 79 (1975)

T.R. Metcalf, K.D. Leka, G. Barnes, B.W. Lites, M.K. Georgoulis, A.A. Pevtsov, K.S. Balasubramaniam, G.A. Gary, J. Jing, J. Li, Y. Liu, H.N. Wang, V. Abramenko, V. Yurchyshyn, Y.-J. Moon, Sol. Phys. 237, 267 (2006)

Y. Mok, Z. Mikić, R. Lionello, J.A. Linker, Astrophys. J. 621, 1098 (2005)

Y. Nakagawa, M.A. Raadu, Sol. Phys. 25, 127 (1972)

V.M. Nakariakov, E. Verwichte, Living Rev. Sol. Phys. 2, 3 (2005)

V.M. Nakariakov, L. Ofman, E.E. Deluca, B. Roberts, J.M. Davila, Science 285, 862 (1999)

A. Nindos, C.E. Alissandrakis, G.B. Gelfreikh, M.R. Kundu, K.P. Dere, A.N. Korzhavin, V.M. Bogod, Sol. Phys. 166, 55 (1996)

A. Nindos, M.R. Kundu, S.M. White, D.E. Gary, K. Shibasaki, K.P. Dere, Astrophys. J. 527, 415 (1999)

A. Nindos, C.E. Alissandrakis, G.B. Gelfreikh, V.M. Bogod, C. Gontikakis, Astron. Astrophys. 386, 658 (2002)

M.D. Papagianis, J.A. Kogut, Air Force Cambridge Res. Lab. Technical Report 75-0430 (1975)

E.N. Parker, Astrophys. J. 330, 474 (1988)

J.H. Piddington, H.C. Minnett, Aust. J. Sci. Res. 4, 131 (1951)

E.R. Priest, T.G. Forbes, Astron. Astrophys. Rev. 10, 313 (2002)

J.A. Ratcliffe, The Magneto-Ionic Theory and its Applications to the Ionosphere (Cambridge University Press, Cambridge, 1959)

R. Rosner, W.H. Tucker, G.S. Vaiana, Astrophys. J. 220, 643 (1978)

B.I. Ryabov, in Solar and Space Weather Radiophysics-Current Status and Future Developments (Kluwer, Dordrecht, 2004), Chap. 7

B.I. Ryabov, N.A. Pilyeva, C.E. Alissandrakis, K. Shibasaki, V.M. Bogod, V.I. Garaimov, G.B. Gelfreikh, Sol. Phys. 185, 157 (1999)

B.I. Ryabov, V.P. Maksimov, S.V. Lesovoi, K. Shibasaki, A. Nindos, A. Pevtsov, Sol. Phys. 226, 223 (2005)

G. Rybicki, A.P. Lightman, Radiative Processes in Astrophysics (Wiley-Interscience, New York, 1979)

T. Sakurai, Publ. Astron. Soc. Jpn. 31, 209 (1979)

T. Sakurai, Sol. Phys. 76, 301 (1982)

A. Schluter, S. Temesvary, Electromagnetic Phenomena in Cosmic Physics, ed. by B. Lenhert. IAU Symp., vol. 6 (1958), p. 263

E.J. Schmahl, M.R. Kundu, K.T. Strong, R.D. Bentley, J.B. Smith, K.R. Krall, Sol. Phys. 80, 233 (1982)

E.J. Schmahl, M.R. Kundu, R.K. Shevgaonkar, D. McConnell, Sol. Phys. 93, 305 (1984)

J.T. Schmelz, G.D. Holman, Adv. Space Res. 11, 109 (1991)

J.T. Schmelz, G.D. Holman, J.W. Brosius, R.D. Gonzalez, Astrophys. J. 399, 733 (1992)

J.T. Schmelz, G.D. Holman, J.W. Brosius, R.F. Willson, Astrophys. J. 434, 786 (1994)

R.K. Shevgaonkar, M.R. Kundu, Astrophys. J. 292, 733 (1985)

K. Shibasaki, Astrophys. J. 550, 1113 (2001)

K. Shibasaki, S. Enome, H. Nakajima, M. Nishio, T. Takano, Y. Hanaoka, C. Torii, H. Sekiguchi, S. Kawashima, T. Bushimata, N. Shinohara, H. Koshiishi, Y. Shiomi, Y. Irimajiri, K.D. Leka, R.C. Canfield, Publ. Astron. Soc. Jpn. 46, L17 (1994) 
K. Shibata, T. Yokoyama, M. Shimojo, in Proceedings of Kofu Symposium, ed. by S. Enome, T. Hirayama. NRO Report No. 360 (1994), p. 75

H. Song, V. Yurchyshyn, G. Yang, C. Tan, W. Chen, H. Wang, Sol. Phys. 238, 141 (2006)

J. Staude, in Third Advances in Solar Physics Euroconference: Magnetic Fields and Oscillations, ed. by B. Schmieder, A. Hofmann, J. Staude. ASP Conference Series, vol. 184 (1999), pp. 113-130

T.H. Stix, The Theory of Plasma Waves (McGraw-Hill, New York, 1962)

K.T. Strong, C.E. Alissandrakis, M.R. Kundu, Astrophys. J. 277, 865 (1984)

S.T. Suess, J.M. Wilcox, J.T. Hoeksema, H. Henning, M. Dryer, J. Geophys. Res. 89, 3957 (1984)

A. Vourlidas, Ph.D. thesis, New Mexico Institute of Mining and Technology, 1996

A. Vourlidas, T.S. Bastian, Astrophys. J. 466, 1039 (1996)

A. Vourlidas, T.S. Bastian, N. Nitta, M.J. Aschwanden, Sol. Phys. 163, 99 (1996)

A. Vourlidas, T.S. Bastian, M.J. Aschwanden, Astrophys. J. 489, 403 (1997)

D.F. Webb, G.D. Holman, J.M. Davis, M.R. Kundu, R.K. Shevgaonkar, Astrophys. J. 315, 716 (1987)

J.M. Wilcox, J.T. Hoeksema, P.H. Scherrer, Science 209, 603 (1980)

S.M. White, in Solar and Space Weather Radiophysics-Current Status and Future Developments (Kluwer, Dordrecht, 2004), Chap. 5

S.M. White, M.R. Kundu, Sol. Phys. 174, 31 (1997)

S.M. White, M.R. Kundu, N. Gopalswamy, Astrophys. J. Suppl. 78, 599 (1992)

S.M. White, M.R. Kundu, N. Gopalswamy, Astrophys. J. Lett. 366, L43 (1991)

S.M. White, R.J. Thomas, J.W. Brosius, M.R. Kundu, Astrophys. J. 534, L203 (2000)

V. Yurchyshyn, Q. Hu, V. Abramenko, Space Weather 3(8), S08C02 (2005)

J. Zhang, N. Gopalswamy, M.R. Kundu, E.J. Schmahl, J.R. Lemen, Sol. Phys. 180, 285 (1998)

V.V. Zheleznyakov, Sov. Astron. 6, 3 (1962)

V.V. Zheleznyakov, Radio Emission of the Sun and the Planets (Pergamon Press, Oxford, 1970)

V.V. Zheleznyakov, E.Y. Zlotnik, Sov. Astron. 7, 485 (1964)

V.V. Zheleznyakov, E.Y. Zlotnik, Sov. Astron. Lett. (TR:PISMA) 14, 73 (1988)

V.V. Zheleznyakov, V.V. Kocharovsky, V.V. Kocharovsky, Astron. Astrophys. 308, 685 (1996)

H. Zirin, B.M. Baumert, G.J. Hurford, Astrophys. J. 370, 779 (1991)

E.Y. Zlotnik, Sov. Astron. 12, 245 (1968a)

E.Y. Zlotnik, Sov. Astron. 12, 464 (1968b)

E.Y. Zlotnik, M.R. Kundu, S.M. White, Radiophys. Quantum Electron. 39, 255 (1996)

E.Y. Zlotnik, S.M. White, M.R. Kundu, in Second Advances in Solar Physics Euroconference: ThreeDimensional Structure of Solar Active Regions, ed. by C.E. Alissandrakis, B. Schmieder. ASP Conf. Series, vol. 155 (1998), p. 135 